Configuration interaction and radiative decay rates in trebly ionized tungsten (W IV)

This article has been downloaded from IOPscience. Please scroll down to see the full text article.

2012 J. Phys. B: At. Mol. Opt. Phys. 45035001

(http://iopscience.iop.org/0953-4075/45/3/035001)

View the table of contents for this issue, or go to the journal homepage for more

Download details:

IP Address: 193.190.193.2

The article was downloaded on 11/01/2012 at 07:47

Please note that terms and conditions apply. 


\title{
Configuration interaction and radiative decay rates in trebly ionized tungsten (W IV)
}

\author{
S Enzonga Yoca ${ }^{1}, \mathbf{P}$ Quinet $^{2,3}$ and É Biémont ${ }^{2,3}$ \\ ${ }^{1}$ Département de Physique, Faculté des Sciences, Université Marien Ngouabi, BP 69 Brazzaville, Congo \\ ${ }^{2}$ Astrophysique et Spectroscopie, Université de Mons - UMONS, B-7000 Mons, Belgium \\ ${ }^{3}$ IPNAS, Université de Liège, B15 Sart Tilman, B-4000 Liège, Belgium \\ E-mail: quinet@umons.ac.be
}

Received 15 November 2011

Published 10 January 2012

Online at stacks.iop.org/JPhysB/45/035001

\begin{abstract}
Radiative decay rates are obtained for the first time for allowed (E1) and forbidden (M1, E2) transitions in trebly ionized tungsten (W IV). Our calculations, motivated by strong interest for low-density plasmas and fusion research, illustrate in a convincing way the importance of core-valence correlation effects which substantially increase the lifetimes and, accordingly, decrease the transition probabilities of this heavy ion. Due to the lack of experimental data for W IV, the reliability of the theoretical ' $f$ ' values can only be tested by comparison of numerical results obtained with two independent (i.e. multiconfiguration Dirac-Fock and relativistic Hartree-Fock with core-polarization effects (HFR+CPOL)) methods well suited for investigating the atomic structure of heavy ions.
\end{abstract}

\section{Introduction}

Atomic data for tungsten ions are of crucial importance in plasma physics in particular in relation with the development of future thermonuclear fusion reactors in which this element will be used as a plasma-facing material (see, e.g., Lipschultz et al 2001, Federici et al 2001, Neu et al 2005, Pospieszczyk 2006, Skinner 2008, 2009). In fusion reactors, tungsten will be sputtered from the wall as a neutral element and the determination of the $\mathrm{W}$ influx rate to the core plasma will depend on a calculation of transport from the wall surface through the scrape-off layer. In that context, the identification of emission lines from all ionization stages of tungsten, including the lowest ones and thus W IV, will greatly aid modelling of the plasma edge and scrape-off layer transport and facilitate the analysis of net tungsten influx rates.

In highly charged tungsten ions, the bulk of lines emitted by hot plasmas can hardly be disentangled and their analysis generally requires global methods (e.g. UTA approaches), while, in lowly or moderately charged ions, the analysis of the individual lines is relevant in spite of the density of the lines and of the complexity of the spectra. A line-by-line analysis of the spectrum emitted by the $\mathrm{W}^{3+}$ ion, and also by the neighbouring ions, is thus in order and further justifies this work.
So far, no radiative decay rates are available for W IV lines and the analysis of the term system of this ion is still very fragmentary. The ground configuration of W IV is $5 \mathrm{~d}^{3}$ with ${ }^{4} F_{3 / 2}$ as the lowest level. The first excited configurations are $5 d^{2} 6 s, 5 d 6 s^{2}, 5 d^{2} 6 p$ and $5 d 6 s 6 p$. All experimentally known energy levels are reported in the compilation of Kramida and Shirai (2009), which is essentially based on the investigations by Iglesias et al (1985) who analysed the W IV spectrum in the region of 60-260 nm using a sliding-spark discharge. These authors identified a total of 774 lines and determined all the 37 levels of the $5 \mathrm{~d}^{3}, 5 \mathrm{~d}^{2} 6 \mathrm{~s}$ and $5 \mathrm{~d} 6 \mathrm{~s}^{2}$ even configurations and all the 68 levels of the $5 d^{2} 6 p$ and $5 d 6 s 6 p$ odd configurations.

We propose, in this paper, a first set of radiative parameters for W IV. This work is an extension of similar investigations carried out recently in W I (Quinet et al 2011), W II (Nilsson et al 2008) and W III (Palmeri et al 2008) ions and as well of a critical evaluation of decay rates for electric dipole and also forbidden transitions in W I, W II and W III (Quinet et al 2010).

\section{Computations}

The ideal way to obtain transition probabilities in an atom or ion is to combine accurate lifetime measurements with 
Table 1. Parameters used in $\mathrm{HFR}_{43}$ calculations.

\begin{tabular}{|c|c|c|c|c|}
\hline Configuration & Parity & $\mathrm{HFR}_{43}$ & Fitted & Fitted/HFR 43 \\
\hline \multicolumn{5}{|c|}{ Even parity } \\
\hline \multirow[t]{6}{*}{$5 d^{3}$} & $E_{\mathrm{av}}$ & & 20749 & \\
\hline & $\mathrm{F}^{2}(5 \mathrm{~d}, 5 \mathrm{~d})$ & 59722 & 50201 & 0.84 \\
\hline & $\mathrm{F}^{4}(5 \mathrm{~d}, 5 \mathrm{~d})$ & 39571 & 33335 & 0.84 \\
\hline & $\alpha$ & - & 19 & - \\
\hline & $\beta$ & - & -499 & - \\
\hline & $\zeta_{5 \mathrm{~d}}$ & 2926 & 2723 & 0.93 \\
\hline \multirow[t]{7}{*}{$5 d^{2} 6 s$} & $E_{\mathrm{av}}$ & - & 50519 & - \\
\hline & $\mathrm{F}^{2}(5 \mathrm{~d}, 5 \mathrm{~d})$ & 61588 & 51743 & 0.84 \\
\hline & $\mathrm{F}^{4}(5 \mathrm{~d}, 5 \mathrm{~d})$ & 40951 & 34881 & 0.85 \\
\hline & $\alpha$ & - & -4 & - \\
\hline & $\beta$ & - & -371 & - \\
\hline & $\zeta_{5 \mathrm{~d}}$ & 3121 & 2918 & 0.94 \\
\hline & $\mathrm{G}^{2}(5 \mathrm{~d}, 6 \mathrm{~s})$ & 21797 & 19824 & 0.91 \\
\hline \multirow[t]{2}{*}{$5 \mathrm{~d} 6 \mathrm{~s}^{2}$} & $E_{\mathrm{av}}$ & & 94113 & \\
\hline & $\zeta_{5 \mathrm{~d}}$ & 3319 & 3111 & 0.94 \\
\hline $5 d^{3}-5 d^{2} 6 s$ & $R^{2}(5 d 5 d, 5 d 6 s)$ & -26715 & -22361 & 0.84 \\
\hline $5 d^{3}-5 d 6 s^{2}$ & $R^{2}(5 d 5 d, 6 s 6 s)$ & 20533 & Fixed & - \\
\hline $5 d^{2} 6 s-5 d 6 s^{2}$ & $R^{2}(5 d 5 d, 5 d 6 s)$ & $\begin{array}{l}-22673 \\
\text { Odd parity }\end{array}$ & Fixed & - \\
\hline \multirow[t]{10}{*}{$5 d^{2} 6 p$} & $E_{\mathrm{av}}$ & - & 112388 & - \\
\hline & $\mathrm{F}^{2}(5 \mathrm{~d}, 5 \mathrm{~d})$ & 62144 & 52522 & 0.85 \\
\hline & $\mathrm{F}^{4}(5 \mathrm{~d}, 5 \mathrm{~d})$ & 41367 & 35263 & 0.85 \\
\hline & $\alpha$ & - & 21 & \\
\hline & $\beta$ & - & -526 & \\
\hline & $\zeta_{5 \mathrm{~d}}$ & 3175 & 3045 & 0.96 \\
\hline & $\zeta_{6 p}$ & 6774 & 7914 & 1.17 \\
\hline & $\mathrm{F}^{2}(5 \mathrm{~d}, 6 \mathrm{p})$ & 26944 & 23274 & 0.86 \\
\hline & $\mathrm{G}^{1}(5 \mathrm{~d}, 6 \mathrm{p})$ & 12263 & 10886 & 0.89 \\
\hline & $\mathrm{G}^{3}(5 \mathrm{~d}, 6 \mathrm{p})$ & 10217 & 7949 & 0.78 \\
\hline \multirow[t]{8}{*}{$5 d 6 s 6 p$} & $E_{\mathrm{av}}$ & - & 152787 & - \\
\hline & $\zeta_{5 \mathrm{~d}}$ & 3370 & 3262 & 0.97 \\
\hline & $\zeta_{6 \mathrm{p}}$ & 7635 & 8751 & 1.15 \\
\hline & $\mathrm{F}^{2}(5 \mathrm{~d}, 6 \mathrm{p})$ & 28171 & 26457 & 0.94 \\
\hline & $\mathrm{G}^{2}(5 \mathrm{~d}, 6 \mathrm{~s})$ & 21614 & 18285 & 0.85 \\
\hline & $\mathrm{G}^{1}(5 \mathrm{~d}, 6 \mathrm{p})$ & 12408 & 11444 & 0.92 \\
\hline & $\mathrm{G}^{3}(5 \mathrm{~d}, 6 \mathrm{p})$ & 10516 & 11961 & 1.14 \\
\hline & $\mathrm{G}^{1}(6 \mathrm{~s}, 6 \mathrm{p})$ & 45053 & 32129 & 0.71 \\
\hline \multirow[t]{3}{*}{$5 d^{2} 6 p-5 d 6 s 6 p$} & $R^{2}(5 d 5 d, 5 d 6 s)$ & -26572 & -22193 (linked) & 0.84 \\
\hline & $R^{2}(5 d 6 p, 6 s 6 p)$ & -23148 & -19333 (linked) & 0.84 \\
\hline & $\mathrm{R}^{1}(5 \mathrm{~d} 6 \mathrm{p}, 6 \mathrm{~s} 6 \mathrm{p})$ & -21353 & -17834 (linked) & 0.84 \\
\hline
\end{tabular}

branching fraction determinations (using experimental or theoretical methods). In practice, however, the experiment is able to provide a limited number of results and needs to be superseded by computational approaches. The reliability of the theoretical models has to be established by comparison with reliable experimental data or with theoretical data obtained in a completely independent way. In the latter case, convergence of the two sets of results helps to assess their reliability. Useful information about the choice of the model can be provided eventually by isoelectronic comparisons with neighbouring ions. This approach was followed here.

In a previous work, in the nearby isoelectronic ion Ta III (Fivet et al 2008), excellent agreement was obtained between HFR+CPOL lifetimes (for a description of the method see Cowan (1981) and Quinet et al (1999)) and the accurate experimental values measured for $5 d^{2} 6 p$ states by the timeresolved laser-induced fluorescence (TR-LIF) spectroscopy. We have adopted the same physical model here considering a set of 43 configurations $\left(5 d^{3}, 5 d^{2} 6 s, 5 d 6 s^{2}, 5 d^{2} 6 d, 5 d 6 p^{2}\right.$, $5 \mathrm{~d} 6 \mathrm{~d}^{2}, 5 \mathrm{~d} 5 \mathrm{f}^{2}, 5 \mathrm{~d} 6 \mathrm{f}^{2}, 5 \mathrm{~d} 6 \mathrm{~s} 6 \mathrm{~d}, 5 \mathrm{~d} 6 \mathrm{p} 5 \mathrm{f}, 5 \mathrm{~d} 6 \mathrm{p} 6 \mathrm{f}, 5 \mathrm{~d} 5 \mathrm{f} 6 \mathrm{f}, 6 \mathrm{~s}^{2} 6 \mathrm{~d}$, $6 \mathrm{~s} 6 \mathrm{p}^{2}, 6 \mathrm{p}^{2} 6 \mathrm{~d}, 6 \mathrm{~s} 6 \mathrm{~d}^{2}, 6 \mathrm{~d}^{3}, 6 \mathrm{~s} 5 \mathrm{f}^{2}, 6 \mathrm{~d} 5 \mathrm{f}^{2}, 6 \mathrm{~s} 6 \mathrm{f}^{2}, 6 \mathrm{~d} 6 \mathrm{f}^{2}$ and $5 \mathrm{~d}^{2} 6 \mathrm{p}$, $5 \mathrm{~d}^{2} 5 \mathrm{f}, 5 \mathrm{~d}^{2} 6 \mathrm{f}, 5 \mathrm{~d} 6 \mathrm{~s} 6 \mathrm{p}, 5 \mathrm{~d} 6 \mathrm{~s} 5 \mathrm{f}, 5 \mathrm{~d} 6 \mathrm{~s} 6 \mathrm{f}, 5 \mathrm{~d} 6 \mathrm{p} 6 \mathrm{~d}, 5 \mathrm{~d} 6 \mathrm{~d} 5 \mathrm{f}, 5 \mathrm{~d} 6 \mathrm{~d} 6 \mathrm{f}$, $6 s^{2} 6 \mathrm{p}, 6 \mathrm{~s}^{2} 5 \mathrm{f}, 6 \mathrm{~s}^{2} 6 \mathrm{f}, 6 \mathrm{p}^{2} 5 \mathrm{f}, 6 \mathrm{p}^{2} 6 \mathrm{f}, 6 \mathrm{p}^{3}, 6 \mathrm{p} 6 \mathrm{~d}^{2}, 6 \mathrm{~d}^{2} 5 \mathrm{f}, 6 \mathrm{~d}^{2} 6 \mathrm{f}$, $6 \mathrm{p} 5 \mathrm{f}^{2}, 6 \mathrm{p} 6 \mathrm{f}^{2}, 5 \mathrm{f}^{2} 6 \mathrm{f}, 5 \mathrm{f} 6 \mathrm{f}^{2}$ ) and this model is referred to as $\mathrm{HFR}_{43}$ throughout this paper.

In the present case, we considered for the corepolarization potential a $4 \mathrm{f}^{14}$ erbium-like core surrounded by three valence electrons. For the dipole polarizability, we adopted the value $\alpha_{d}=2.50 a_{0}^{3}$, which corresponds to a W VII ionic core and was found by considering the behaviour of $\alpha_{d}$ along the erbium isoelectronic sequence (Fraga et al 1976). The cut-off radius used was the HFR mean radius of the outermost core orbital 5 p, i.e. $r_{c}=1.18 a_{0}$.

The $a b$ initio calculated energy levels are generally not close enough to the experimental ones and a leastsquares fitting adjustment of the average energies $\left(E_{\mathrm{av}}\right)$, Slater 
Table 2. Comparison of radiative lifetimes computed in this work for odd-parity levels of W IV.

\begin{tabular}{|c|c|c|c|c|c|}
\hline \multirow[b]{2}{*}{ Level $^{\mathrm{a}}$} & \multirow{2}{*}{$\begin{array}{l}\text { Energy }^{\mathrm{a}} \\
\left(\mathrm{cm}^{-1}\right)\end{array}$} & \multicolumn{4}{|c|}{ Lifetime (ns) } \\
\hline & & $\mathrm{MCDF}_{16}$ & $\mathrm{HFR}_{16}$ & $\mathrm{HFR}_{43}$ & $\mathrm{HFR}_{43}+\mathrm{CPOL}$ \\
\hline $5 d^{2}\left({ }^{3} F\right) 6 p{ }^{4} G_{5 / 2}^{\circ}$ & 85289.99 & 1.06 & 1.06 & 1.16 & 1.39 \\
\hline $5 d^{2}\left({ }^{3} F\right) 6 p^{4} F_{3 / 2}^{\circ}$ & 89893.06 & 0.42 & 0.45 & 0.49 & 0.56 \\
\hline $5 d^{2}\left({ }^{3} F\right) 6 p{ }^{4} G_{7 / 2}^{\circ}$ & 92845.71 & 0.84 & 0.84 & 0.92 & 1.10 \\
\hline $5 d^{2}\left({ }^{3} \mathrm{~F}\right) 6 \mathrm{p}^{4} \mathrm{~F}_{5 / 2}^{\circ}$ & 94525.28 & 0.51 & 0.50 & 0.55 & 0.63 \\
\hline $5 d^{2}\left({ }^{3} P\right) 6 p{ }^{4} D_{1 / 2}^{\circ}$ & 98463.71 & 0.27 & 0.34 & 0.35 & 0.40 \\
\hline $5 d^{2}\left({ }^{3} P\right) 6 p{ }^{4} D_{5 / 2}^{\circ}$ & 98691.76 & 0.45 & 0.52 & 0.54 & 0.64 \\
\hline $5 d^{2}\left({ }^{3} F\right) 6 p^{4} G_{9 / 2}^{\circ}$ & 99028.26 & 0.68 & 0.64 & 0.69 & 0.81 \\
\hline $5 d^{2}\left({ }^{1} \mathrm{D}\right) 6 \mathrm{p}^{2} \mathrm{D}_{3 / 2}^{\circ}$ & 99867.18 & 0.45 & 0.48 & 0.54 & 0.63 \\
\hline $5 d^{2}\left({ }^{3} F\right) 6 p{ }^{4} F_{7 / 2}^{\circ}$ & 100322.36 & 0.38 & 0.41 & 0.45 & 0.51 \\
\hline $5 d^{2}\left({ }^{3} F\right) 6 p^{2} D_{3 / 2}^{\circ}$ & 100449.92 & 0.30 & 0.32 & 0.34 & 0.39 \\
\hline $5 d^{2}\left({ }^{3} P\right) 6 p^{2} S_{1 / 2}^{o j / 2}$ & 101275.42 & 0.36 & 0.39 & 0.40 & 0.47 \\
\hline $5 d^{2}\left({ }^{3} F\right) 6 p^{2} F_{7 / 2}^{\circ}$ & 102867.63 & 0.36 & 0.31 & 0.34 & 0.39 \\
\hline $5 d^{2}\left({ }^{3} F\right) 6 p^{4} D_{5 / 2}^{\circ}$ & 103216.51 & 0.28 & 0.28 & 0.30 & 0.35 \\
\hline $5 d^{2}\left({ }^{3} F\right) 6 p{ }^{4} D_{1 / 2}^{\circ / 2}$ & 103682.37 & 0.69 & 0.47 & 0.52 & 0.61 \\
\hline $5 d^{2}\left({ }^{3} P\right) 6 p{ }^{4} D_{3 / 2}^{\circ}$ & 103952.54 & 0.31 & 0.35 & 0.38 & 0.43 \\
\hline $5 d^{2}\left({ }^{3} F\right) 6 p{ }^{4} F_{9 / 2}^{\circ}$ & 105263.40 & 0.38 & 0.44 & 0.47 & 0.56 \\
\hline $5 d^{2}\left({ }^{3} F\right) 6 p^{2} G_{7 / 2}^{\circ}$ & 106730.40 & 0.35 & 0.38 & 0.41 & 0.47 \\
\hline $5 d^{2}\left({ }^{3} F\right) 6 p^{2} F_{5 / 2}^{\circ}$ & 106754.40 & 0.45 & 0.47 & 0.51 & 0.60 \\
\hline $5 d^{2}\left({ }^{1} G\right) 6 p^{2} G_{7 / 2}^{\circ}$ & 108871.96 & 0.34 & 0.43 & 0.46 & 0.53 \\
\hline $5 d^{2}\left({ }^{3} F\right) 6 p^{4} D_{3 / 2}^{\circ}$ & 108922.34 & 0.54 & 0.41 & 0.46 & 0.54 \\
\hline $5 d^{2}\left({ }^{3} F\right) 6 p^{4} G_{11 / 2}^{\circ}$ & 109190.30 & 0.65 & 0.61 & 0.66 & 0.80 \\
\hline $5 d^{2}\left({ }^{3} P\right) 6 p{ }^{4} S_{3 / 2}^{\circ}$ & 109882.34 & 0.30 & 0.34 & 0.38 & 0.43 \\
\hline $5 d^{2}\left({ }^{1} \mathrm{G}\right) 6 p^{2} \mathrm{H}_{9 / 2}^{\circ}$ & 110252.75 & 0.40 & 0.43 & 0.46 & 0.54 \\
\hline $5 \mathrm{~d}^{2}\left({ }^{1} \mathrm{D}\right) 6 \mathrm{p}^{2} \mathrm{~F}_{5 / 2}^{\circ}$ & 110658.47 & 0.28 & 0.27 & 0.30 & 0.34 \\
\hline $5 d^{2}\left({ }^{3} F\right) 6 p^{4} D_{7 / 2}^{\circ}$ & 111515.16 & 0.23 & 0.24 & 0.27 & 0.30 \\
\hline $5 d^{2}\left({ }^{3} P\right) 6 p^{4} P_{1 / 2}^{\circ}$ & 112861.20 & 0.32 & 0.33 & 0.36 & 0.42 \\
\hline $5 d^{2}\left({ }^{3} P\right) 6 p^{4} P_{3 / 2}^{\circ}$ & 113533.11 & 0.37 & 0.37 & 0.40 & 0.47 \\
\hline $5 d^{2}\left({ }^{3} F\right) 6 p^{2} D_{5 / 2}^{\circ}$ & 113620.96 & 0.38 & 0.42 & 0.46 & 0.54 \\
\hline $5 d^{2}\left({ }^{3} P\right) 6 p^{4} P_{5 / 2}^{\circ}$ & 115317.71 & 0.46 & 0.47 & 0.48 & 0.56 \\
\hline $5 d^{2}\left({ }^{3} F\right) 6 p^{2} G_{9 / 2}^{\circ}$ & 115422.93 & 0.27 & 0.23 & 0.26 & 0.29 \\
\hline $5 d^{2}\left({ }^{3} P\right) 6 p^{4} D_{7 / 2}^{\circ}$ & 116110.43 & 0.36 & 0.34 & 0.36 & 0.43 \\
\hline $5 d^{2}\left({ }^{1} \mathrm{D}\right) 6 \mathrm{p}^{2} \mathrm{P}_{3 / 2}^{\circ}$ & 116421.86 & 0.31 & 0.32 & 0.34 & 0.39 \\
\hline $5 d^{2}\left({ }^{1} \mathrm{D}\right) 6 \mathrm{p}^{2} \mathrm{P}_{1 / 2}^{\circ}$ & 117725.20 & 0.31 & 0.35 & 0.40 & 0.46 \\
\hline $5 d^{2}\left({ }^{3} P\right) 6 p^{2} D_{3 / 2}^{\circ}$ & 119041.11 & 0.31 & 0.28 & 0.32 & 0.36 \\
\hline $5 \mathrm{~d}^{2}\left({ }^{1} \mathrm{D}\right) 6 \mathrm{p}^{2} \mathrm{D}_{5 / 2}^{\circ}$ & 119079.04 & 0.43 & 0.34 & 0.39 & 0.44 \\
\hline $5 d^{2}\left({ }^{1} G\right) 6 p^{2} G_{9 / 2}^{\circ / 2}$ & 119694.03 & 0.28 & 0.33 & 0.37 & 0.43 \\
\hline $5 \mathrm{~d}^{2}\left({ }^{1} \mathrm{D}\right) 6 \mathrm{p}^{2} \mathrm{~F}_{7 / 2}^{\circ}$ & 121644.05 & 0.23 & 0.27 & 0.28 & 0.33 \\
\hline $5 d^{2}\left({ }^{3} \mathrm{P}\right) 6 \mathrm{p}^{2} \mathrm{P}_{1 / 2}^{\circ}$ & 121677.79 & 0.41 & 0.36 & 0.36 & 0.42 \\
\hline $5 d^{2}\left({ }^{1} \mathrm{G}\right) 6 \mathrm{p}^{2} \mathrm{H}_{11 / 2}^{\circ}$ & 122847.40 & 0.47 & 0.45 & 0.49 & 0.58 \\
\hline $5 d^{2}\left({ }^{3} \mathrm{P}\right) 6 \mathrm{p}^{2} \mathrm{D}_{5 / 2}^{\circ}$ & 123216.93 & 0.32 & 0.37 & 0.41 & 0.48 \\
\hline $5 d^{2}\left({ }^{1} G\right) 6 p^{2} F_{7 / 2}^{\circ}$ & 123908.20 & 0.34 & 0.32 & 0.36 & 0.42 \\
\hline $5 \mathrm{~d}^{2}\left({ }^{3} \mathrm{P}\right) 6 \mathrm{p}^{2} \mathrm{P}_{3 / 2}^{\circ}$ & 126076.70 & 0.38 & 0.35 & 0.40 & 0.46 \\
\hline $5 d^{2}\left({ }^{1} G\right) 6 p^{2} F_{5 / 2}^{\circ}$ & 127077.24 & 0.37 & 0.35 & 0.38 & 0.45 \\
\hline $5 \mathrm{~d}\left({ }^{2} \mathrm{D}\right) 6 \mathrm{~s} 6 \mathrm{p}\left({ }^{3} \mathrm{P}^{\circ}\right){ }^{4} \mathrm{~F}_{3 / 2}^{\circ}$ & 130118.60 & 0.52 & 0.71 & 0.72 & 0.83 \\
\hline $5 \mathrm{~d}^{2}\left({ }^{1} \mathrm{~S}\right) 6 \mathrm{p}{ }^{2} \mathrm{P}_{1 / 2}^{\circ}$ & 133358.14 & 0.32 & 0.31 & 0.32 & 0.37 \\
\hline $5 \mathrm{~d}\left({ }^{2} \mathrm{D}\right) 6 \mathrm{~s} 6 \mathrm{p}\left({ }^{3} \mathrm{P}^{\circ}\right){ }^{4} \mathrm{~F}_{5 / 2}^{\circ}$ & 133785.47 & 0.47 & 0.63 & 0.64 & 0.74 \\
\hline $5 \mathrm{~d}\left({ }^{2} \mathrm{D}\right) 6 \mathrm{~s} 6 \mathrm{p}\left({ }^{3} \mathrm{P}^{\circ}\right){ }^{4} \mathrm{D}_{1 / 2}^{\circ}$ & 134379.44 & 0.24 & 0.30 & 0.34 & 0.39 \\
\hline $5 \mathrm{~d}\left({ }^{2} \mathrm{D}\right) 6 \mathrm{~s} 6 \mathrm{p}\left({ }^{3} \mathrm{P}^{\circ}\right){ }^{4} \mathrm{D}_{3 / 2}^{\circ}$ & 135823.21 & 0.27 & 0.34 & 0.35 & 0.40 \\
\hline $5 \mathrm{~d}\left({ }^{2} \mathrm{D}\right) 6 \mathrm{~s} 6 \mathrm{p}\left({ }^{3} \mathrm{P}^{\circ}\right){ }^{4} \mathrm{D}_{5 / 2}^{\circ}$ & 140317.03 & 0.27 & 0.33 & 0.34 & 0.39 \\
\hline $5 \mathrm{~d}\left({ }^{2} \mathrm{D}\right) 6 \mathrm{~s} 6 \mathrm{p}\left({ }^{3} \mathrm{P}^{\circ}\right){ }^{4} \mathrm{~F}_{7 / 2}^{\circ}$ & 140788.56 & 0.48 & 0.55 & 0.55 & 0.63 \\
\hline $5 \mathrm{~d}^{2}\left({ }^{1} \mathrm{~S}\right) 6 \mathrm{p}{ }^{2} \mathrm{P}_{1 / 2}^{\circ}$ & 142203.49 & 0.38 & 0.33 & 0.36 & 0.42 \\
\hline $5 \mathrm{~d}\left({ }^{2} \mathrm{D}\right) 6 \mathrm{~s} 6 \mathrm{p}\left({ }^{3} \mathrm{P}^{\circ}\right){ }^{2} \mathrm{D}_{5 / 2}^{\circ}$ & 143533.47 & 0.32 & 0.42 & 0.43 & 0.49 \\
\hline $5 \mathrm{~d}\left({ }^{2} \mathrm{D}\right) 6 \mathrm{~s} 6 \mathrm{p}\left({ }^{3} \mathrm{P}^{\circ}\right){ }^{4} \mathrm{P}_{3 / 2}^{\circ}$ & 145569.33 & 0.31 & 0.42 & 0.42 & 0.49 \\
\hline $5 \mathrm{~d}\left({ }^{2} \mathrm{D}\right) 6 \mathrm{~s} 6 \mathrm{p}\left({ }^{3} \mathrm{P}^{\circ}\right){ }^{4} \mathrm{D}_{7 / 2}^{\circ}$ & 146205.65 & 0.29 & 0.33 & 0.35 & 0.40 \\
\hline $5 \mathrm{~d}\left({ }^{2} \mathrm{D}\right) 6 \mathrm{~s} 6 \mathrm{p}\left({ }^{3} \mathrm{P}^{\circ}\right){ }^{2} \mathrm{D}_{3 / 2}^{\circ}$ & 146750.22 & 0.30 & 0.36 & 0.39 & 0.44 \\
\hline $5 \mathrm{~d}\left({ }^{2} \mathrm{D}\right) 6 \mathrm{~s} 6 \mathrm{p}\left({ }^{3} \mathrm{P}^{\circ}\right){ }^{4} \mathrm{P}_{1 / 2}^{\circ}$ & 147405.37 & 0.36 & 0.41 & 0.42 & 0.48 \\
\hline $5 \mathrm{~d}\left({ }^{2} \mathrm{D}\right) 6 \mathrm{~s} 6 \mathrm{p}\left({ }^{3} \mathrm{P}^{\circ}\right){ }^{4} \mathrm{P}_{5 / 2}^{\circ}$ & 149758.30 & 0.32 & 0.40 & 0.40 & 0.46 \\
\hline $5 \mathrm{~d}\left({ }^{2} \mathrm{D}\right) 6 \mathrm{~s} 6 \mathrm{p}\left({ }^{3} \mathrm{P}^{\circ}\right){ }^{4} \mathrm{~F}_{9 / 2}^{\circ}$ & 150422.00 & 0.75 & 0.79 & 0.76 & 0.87 \\
\hline
\end{tabular}


Table 2. (Continued.)

\begin{tabular}{llllll}
\hline & $\begin{array}{l}\text { Energy } \\
\end{array}$ & \multicolumn{4}{c}{ Lifetime $\left.^{\mathrm{a}} \mathrm{ns}\right)$} \\
\cline { 3 - 6 } Level $^{\mathrm{a}}$ & $\left(\mathrm{cm}^{-1}\right)$ & $\mathrm{MCDF}_{16}$ & $\mathrm{HFR}_{16}$ & $\mathrm{HFR}_{43}$ & $\mathrm{HFR}_{43}+\mathrm{CPOL}$ \\
\hline $5 \mathrm{~d}\left({ }^{2} \mathrm{D}\right) 6 \mathrm{~s} 6 \mathrm{p}\left({ }^{3} \mathrm{P}^{\circ}\right){ }^{2} \mathrm{~F}_{5 / 2}^{\circ}$ & 155752.99 & 0.29 & 0.35 & 0.35 & 0.40 \\
$5 \mathrm{~d}\left({ }^{2} \mathrm{D}\right) 6 \mathrm{~s} 6 \mathrm{p}\left({ }^{3} \mathrm{P}^{\circ}\right){ }^{2} \mathrm{P}_{3 / 2}^{\circ}$ & 157726.0 & 0.32 & 0.23 & 0.24 & 0.29 \\
$5 \mathrm{~d}\left({ }^{2} \mathrm{D}\right) 6 \mathrm{~s} 6 \mathrm{p}\left({ }^{3} \mathrm{P}^{\circ}\right){ }^{2} \mathrm{~F}_{7 / 2}^{\circ}$ & 157984.23 & 0.24 & 0.26 & 0.27 & 0.30 \\
$5 \mathrm{~d}\left({ }^{2} \mathrm{D}\right) 6 \mathrm{~s} 6 \mathrm{p}\left({ }^{3} \mathrm{P}^{\circ}\right){ }^{2} \mathrm{P}_{1 / 2}^{\circ}$ & 162651.8 & 0.35 & 0.34 & 0.39 & 0.44 \\
$5 \mathrm{~d}\left({ }^{2} \mathrm{D}\right) 6 \mathrm{~s} 6 \mathrm{p}\left({ }^{1} \mathrm{P}^{\circ}\right){ }^{2} \mathrm{~F}_{5 / 2}^{\circ}$ & 163375.42 & 0.15 & 0.14 & 0.15 & 0.18 \\
$5 \mathrm{~d}\left({ }^{2} \mathrm{D}\right) 6 \mathrm{~s} 6 \mathrm{p}\left({ }^{1} \mathrm{P}^{\circ}\right){ }^{2} \mathrm{D}_{3 / 2}^{\circ}$ & 163536.02 & 0.12 & 0.18 & 0.17 & 0.20 \\
$5 \mathrm{~d}\left({ }^{2} \mathrm{D}\right) 6 \mathrm{~s} 6 \mathrm{p}\left({ }^{1} \mathrm{P}^{\circ}\right){ }^{2} \mathrm{P}_{1 / 2}^{\circ}$ & 168767.50 & 0.17 & 0.17 & 0.16 & 0.20 \\
$5 \mathrm{~d}\left({ }^{2} \mathrm{D}\right) 6 \mathrm{~s} 6 \mathrm{p}\left({ }^{1} \mathrm{P}^{\circ}\right){ }^{2} \mathrm{D}_{5 / 2}^{\circ}$ & 169912.91 & 0.12 & 0.13 & 0.12 & 0.15 \\
$5 \mathrm{~d}\left({ }^{2} \mathrm{D}\right) 6 \mathrm{~s} 6 \mathrm{p}\left({ }^{1} \mathrm{P}^{\circ}\right){ }^{2} \mathrm{~F}_{7 / 2}^{\circ}$ & 171306.23 & 0.16 & 0.17 & 0.18 & 0.22 \\
$5 \mathrm{~d}\left({ }^{2} \mathrm{D}\right) 6 \mathrm{~s} 6 \mathrm{p}\left({ }^{1} \mathrm{P}^{\circ}\right){ }^{2} \mathrm{P}_{3 / 2}^{\circ}$ & 174786.10 & 0.15 & 0.15 & 0.14 & 0.17 \\
\hline
\end{tabular}

${ }^{a}$ Level designations and energies from Kramida and Shirai (2009).

integrals $\left(F^{k}, G^{k}, R^{k}\right)$, spin-orbit integrals $\left(\zeta_{n l}\right)$ and effective interaction parameters $(\alpha, \beta)$ is required. For that purpose, the experimental levels compiled by Kramida and Shirai (2009) were considered. Thus, 37 even levels belonging to the $5 \mathrm{~d}^{3}, 5 \mathrm{~d}^{2} 6 \mathrm{~s}$ and $5 \mathrm{~d} 6 \mathrm{~s}^{2}$ configurations were fitted with 16 variable parameters, while 68 odd levels belonging to the $5 d^{2} 6 p$ and $5 d 6 s 6 p$ configurations were fitted with 19 adjustable parameters. The optimized values of these parameters are given in table 1 . The mean deviations in the fitting procedure were found to be equal to $40 \mathrm{~cm}^{-1}$ for the even parity and $159 \mathrm{~cm}^{-1}$ for the odd parity. The difference between the mean deviations is essentially due to the fact that oddparity states are much more affected by intermediate coupling and configuration interaction effects than even-parity levels. Indeed, it was found that the calculated average purity of the wavefunctions in the $L S$ coupling for $5 \mathrm{~d}^{3}, 5 \mathrm{~d}^{2} 6 \mathrm{~s}$ and $5 \mathrm{~d} 6 \mathrm{~s}^{2}$ even levels was equal to $75 \%$, while the corresponding value for $5 d^{2} 6 p$ and $5 d 6 s 6 p$ odd levels only reached $50 \%$.

The reliability of the HFR+CPOL method (based on a perturbational treatment of relativistic effects including the spin-orbit, mass-velocity and Darwin one-body relativistic corrections in the Hartree-Fock equations) was established by comparing the radiative parameters obtained using this approximation with the relativistic multiconfiguration DiracFock (MCDF) approach in the form described by Grant et al (1980) and McKenzie et al (1980). To do so, the latest version of the general-purpose relativistic atomic structure package (GRASP) developed by Norrington (2009) was used in the extended average level (EAL) mode. Relativistic twobody Breit interaction and quantum electrodynamics (QED) corrections such as self-energy and vacuum polarization were included in the calculations, while nuclear effects were estimated by considering a uniform charge distribution with the usual atomic weight of tungsten, i.e. $183.85 \mathrm{amu}$. The non-relativistic configurations retained in the MCDF model were chosen amongst those of the above $\mathrm{HFR}_{43}$ model having the strongest interactions with the $5 \mathrm{~d}^{3}, 5 \mathrm{~d}^{2} 6 \mathrm{~s}, 5 \mathrm{~d} 6 \mathrm{~s}^{2}, 5 \mathrm{~d}^{2} 6 \mathrm{p}$ and 5d6s6p configurations. More precisely, the following 16 configurations were included in the multiconfiguration expansion: $5 \mathrm{~d}^{3}, 5 \mathrm{~d}^{2} 6 \mathrm{~s}, 5 \mathrm{~d} 6 \mathrm{~s}^{2}, 5 \mathrm{~d}^{2} 6 \mathrm{~d}, 5 \mathrm{~d} 6 \mathrm{p}^{2}, 5 \mathrm{~d} 6 \mathrm{~d}^{2}, 5 \mathrm{~d} 6 \mathrm{~s} 6 \mathrm{~d}$, $6 s^{2} 6 \mathrm{~d}, 6 \mathrm{~s} 6 \mathrm{p}^{2}, 6 \mathrm{~s} 6 \mathrm{~d}^{2}$ (even parity) and $5 \mathrm{~d}^{2} 6 \mathrm{p}, 5 \mathrm{~d} 6 \mathrm{~s} 6 \mathrm{p}, 5 \mathrm{~d} 6 \mathrm{p} 6 \mathrm{~d}$, $6 s^{2} 6 \mathrm{p}, 6 \mathrm{p}^{3}, 6 \mathrm{p} 6 \mathrm{~d}^{2}$ (odd parity). Moreover, in this calculation (referred to as $\mathrm{MCDF}_{16}$ ), transition energies were replaced by experimental values when computing transition probabilities. It was verified that $\mathrm{MCDF}_{16}$ results, obtained in both the length (Babushkin) and velocity (Coulomb) gauges, were in rather good agreement, the mean ratio $A_{\text {velocity }} / A_{\text {length }}$ being found equal to 0.93 (for $A \geqslant 10^{9} \mathrm{~s}^{-1}$ ), 1.07 (for $A \geqslant 10^{8}$ $\mathrm{s}^{-1}$ ) and 1.10 (for $A \geqslant 10^{7} \mathrm{~s}^{-1}$ ). As a consequence, only the length form results will be considered throughout the paper. In order to make a significant comparison, the same set of 16 configurations was included in a HFR model $\left(\mathrm{HFR}_{16}\right)$ in which a semi-empirical adjustment of the radial parameters was performed in the same way as the one carried out in the extended $\mathrm{HFR}_{43}$ model described above.

\section{Results}

In table 2, we compare the radiative lifetimes computed using our different approaches for all experimentally known oddparity levels. It is worth noting that $\mathrm{MCDF}_{16}$ and $\mathrm{HFR}_{16}$ values are in good agreement (within $\sim 10-20 \%$ ) for most levels, the only exceptions occurring for the $5 d^{2} 6 p$ levels at 103682.37 and $108922.34 \mathrm{~cm}^{-1}$ and the 5d6s6p levels at $130118.60,133785.47,143533.47,145569.33$ and 157726.0 $\mathrm{cm}^{-1}$ for which larger discrepancies (up to 30-40\%) can be observed. This overall good agreement between MCDF and HFR methods used with the same limited set of configurations gives solid support to HFR theoretical results obtained using more extended multiconfiguration expansion and including core-polarization effects. The importance of these latter effects are clearly illustrated in table 2 , where $\mathrm{HFR}_{43}+\mathrm{CPOL}$ lifetimes appear systematically $15-20 \%$ longer than $\mathrm{HFR}_{43}$ values, while $\mathrm{HFR}_{16}$ and $\mathrm{HFR}_{43}$ results in general do not differ by more than a few per cent.

Transition probabilities $(g A)$ and oscillator strengths (log $g f$ ) calculated using the $\mathrm{HFR}_{43}+\mathrm{CPOL}$ method described above are reported in table 3 for a set of W IV lines ranging from 93 to $238 \mathrm{~nm}$. Only transitions involving energy levels below $140000 \mathrm{~cm}^{-1}$ and with $\log g f \geqslant$ -1 are listed in the table. A more detailed table is available in our database on sixth-row elements (DESIRE) at http://w3.umons.ac.be/astro/desire.shtml. 
Table 3. Oscillator strengths ( $\log g f$ ) and transition probabilities $(g A)$ for W IV lines. Only transitions involving energy levels below $140000 \mathrm{~cm}^{-1}$ and with $\log g f \geqslant-1$ are listed. $A(B)$ stands for $A \times 10^{B}$.

\begin{tabular}{|c|c|c|c|c|c|c|c|c|}
\hline \multirow[b]{2}{*}{$\lambda^{\mathrm{a}}(\mathrm{nm})$} & \multicolumn{3}{|c|}{ Lower level $^{\mathrm{a}}$} & \multicolumn{3}{|c|}{ Upper level $^{\mathrm{a}}$} & \multirow[b]{2}{*}{$\log g f^{\mathrm{d}}$} & \multirow[b]{2}{*}{$g A^{\mathrm{d}}\left(\mathrm{s}^{-1}\right)$} \\
\hline & $E\left(\mathrm{~cm}^{-1}\right)$ & Configuration & $J$ & $E\left(\mathrm{~cm}^{-1}\right)$ & Configuration & $J$ & & \\
\hline 93.5852 & 9256.39 & $5 d^{3}$ & $9 / 2$ & 116110.43 & $5 d^{2} 6 p$ & $7 / 2$ & -0.63 & $1.81(9)$ \\
\hline 94.1917 & 9256.39 & $5 d^{3}$ & $9 / 2$ & 115422.93 & $5 d^{2} 6 p$ & $9 / 2$ & -0.74 & $1.37(9)$ \\
\hline 94.8412 & 16204.85 & $5 d^{3}$ & $9 / 2$ & 121644.05 & $5 d^{2} 6 p$ & $7 / 2$ & -0.83 & $1.09(9)$ \\
\hline 96.2334 & 6744.63 & $5 d^{3}$ & $7 / 2$ & 110658.47 & $5 d^{2} 6 p$ & $5 / 2$ & -0.84 & $1.05(9)$ \\
\hline 96.7961 & 22766.37 & $5 d^{3}$ & $5 / 2$ & 126076.70 & $5 d^{2} 6 p$ & $3 / 2$ & -0.99 & $7.21(8)$ \\
\hline 96.9379 & 18519.01 & $5 d^{3}$ & $3 / 2$ & 121677.79 & $5 d^{2} 6 p$ & $1 / 2$ & -0.78 & $1.18(9)$ \\
\hline 98.1462 & 17189.88 & $5 d^{3}$ & $5 / 2$ & 119079.04 & $5 d^{2} 6 p$ & $5 / 2$ & -0.97 & $7.42(8)$ \\
\hline 98.7410 & 0.00 & $5 d^{3}$ & $3 / 2$ & 101275.42 & $5 d^{2} 6 p$ & $1 / 2$ & -0.95 & 7.63(8) \\
\hline 98.9187 & 32692.40 & $5 d^{2} 6 s$ & $3 / 2$ & 133785.47 & $5 d 6 s 6 p$ & $5 / 2$ & -0.90 & $8.62(8)$ \\
\hline $99.0135^{b}$ & 9256.39 & $5 d^{3}$ & $9 / 2$ & 110252.75 & $5 d^{2} 6 p$ & $9 / 2$ & -0.77 & $1.17(9)$ \\
\hline 99.0995 & 25167.81 & $5 d^{3}$ & $3 / 2$ & 126076.70 & $5 d^{2} 6 p$ & $3 / 2$ & -0.95 & $7.56(8)$ \\
\hline 99.2760 & 35093.78 & $5 d^{2} 6 s$ & $5 / 2$ & 135823.21 & $5 \mathrm{~d} 6 \mathrm{~s} 6 \mathrm{p}$ & $3 / 2$ & 0.05 & $7.57(9)$ \\
\hline 99.3795 & 22223.06 & $5 d^{3}$ & $11 / 2$ & 122847.40 & $5 d^{2} 6 p$ & $11 / 2$ & 0.11 & $8.63(9)$ \\
\hline $99.5519^{c}$ & 22766.37 & $5 d^{3}$ & $5 / 2$ & 123216.93 & $5 d^{2} 6 p$ & $5 / 2$ & -0.79 & $1.09(9)$ \\
\hline 99.5869 & 3537.49 & $5 d^{3}$ & $5 / 2$ & 103952.54 & $5 d^{2} 6 p$ & $3 / 2$ & -0.42 & $2.52(9)$ \\
\hline 100.0142 & 6744.63 & $5 d^{3}$ & $7 / 2$ & 106730.40 & $5 d^{2} 6 p$ & $7 / 2$ & -0.55 & $1.86(9)$ \\
\hline 100.0664 & 9256.39 & $5 d^{3}$ & $9 / 2$ & 109190.30 & $5 d^{2} 6 p$ & $11 / 2$ & -0.63 & $1.56(9)$ \\
\hline 100.1883 & 24096.03 & $5 d^{3}$ & $9 / 2$ & 123908.20 & $5 d^{2} 6 p$ & $7 / 2$ & -0.21 & $4.15(9)$ \\
\hline 100.3860 & 9256.39 & $5 d^{3}$ & $9 / 2$ & 108871.96 & $5 d^{2} 6 p$ & $7 / 2$ & -0.23 & $3.94(9)$ \\
\hline 100.8681 & 27937.63 & $5 d^{3}$ & $5 / 2$ & 127077.24 & $5 d^{2} 6 p$ & $5 / 2$ & -0.28 & $3.43(9)$ \\
\hline 101.0914 & 17189.88 & $5 d^{3}$ & $5 / 2$ & 116110.43 & $5 d^{2} 6 p$ & $7 / 2$ & -0.61 & $1.59(9)$ \\
\hline 101.1354 & 22766.37 & $5 d^{3}$ & $5 / 2$ & 121644.05 & $5 d^{2} 6 p$ & $7 / 2$ & -0.90 & $8.19(8)$ \\
\hline $101.3254^{\mathrm{c}}$ & 35093.78 & $5 d^{2} 6 s$ & $5 / 2$ & 133785.47 & $5 d 6 s 6 p$ & $5 / 2$ & -0.03 & $6.04(9)$ \\
\hline 101.5604 & 0.00 & $5 d^{3}$ & $3 / 2$ & 98463.71 & $5 d^{2} 6 p$ & $1 / 2$ & -0.32 & $3.08(9)$ \\
\hline 101.6677 & 15260.92 & $5 d^{3}$ & $7 / 2$ & 113620.96 & $5 d^{2} 6 p$ & $5 / 2$ & -0.93 & $7.57(8)$ \\
\hline 101.8965 & 27937.63 & $5 d^{3}$ & $5 / 2$ & 126076.70 & $5 d^{2} 6 p$ & $3 / 2$ & -0.86 & $8.94(8)$ \\
\hline 101.9082 & 17189.88 & $5 d^{3}$ & $5 / 2$ & 115317.71 & $5 d^{2} 6 p$ & $5 / 2$ & -0.36 & $2.82(9)$ \\
\hline 101.9897 & 25167.81 & $5 d^{3}$ & $3 / 2$ & 123216.93 & $5 d^{2} 6 p$ & $5 / 2$ & -0.98 & $6.71(8)$ \\
\hline 102.5137 & 24096.03 & $5 d^{3}$ & $9 / 2$ & 121644.05 & $5 d^{2} 6 p$ & $7 / 2$ & 0.30 & $1.27(10)$ \\
\hline 102.5948 & 22223.06 & $5 d^{3}$ & $11 / 2$ & 119694.03 & $5 d^{2} 6 p$ & $9 / 2$ & -0.08 & $5.27(9)$ \\
\hline 102.6419 & 32692.40 & $5 d^{2} 6 s$ & $3 / 2$ & 130118.60 & $5 d 6 s 6 p$ & $3 / 2$ & -0.17 & $4.33(9)$ \\
\hline 103.1859 & 3537.49 & $5 d^{3}$ & $5 / 2$ & 100449.92 & $5 d^{2} 6 p$ & $3 / 2$ & -0.15 & 4.44(9) \\
\hline 103.5910 & 10220.65 & $5 d^{3}$ & $3 / 2$ & 106754.40 & $5 d^{2} 6 p$ & $5 / 2$ & -0.89 & $7.96(8)$ \\
\hline 103.6163 & 25167.81 & $5 d^{3}$ & $3 / 2$ & 121677.79 & $5 d^{2} 6 p$ & $1 / 2$ & -0.49 & $2.03(9)$ \\
\hline 103.6570 & 6744.63 & $5 d^{3}$ & $7 / 2$ & 103216.51 & $5 d^{2} 6 p$ & $5 / 2$ & 0.19 & $9.63(9)$ \\
\hline 103.7952 & 17189.88 & $5 d^{3}$ & $5 / 2$ & 113533.11 & $5 d^{2} 6 p$ & $3 / 2$ & -0.24 & $3.58(9)$ \\
\hline 104.1589 & 9256.39 & $5 d^{3}$ & $9 / 2$ & 105263.40 & $5 d^{2} 6 p$ & $9 / 2$ & 0.09 & 7.53(9) \\
\hline 104.6044 & 24096.03 & $5 d^{3}$ & $9 / 2$ & 119694.03 & $5 d^{2} 6 p$ & $9 / 2$ & 0.11 & $7.87(9)$ \\
\hline 104.7165 & 28412.13 & $5 d^{3}$ & $7 / 2$ & 123908.20 & $5 d^{2} 6 p$ & $7 / 2$ & -0.23 & $3.59(9)$ \\
\hline 104.8244 & 15260.92 & $5 d^{3}$ & $7 / 2$ & 110658.47 & $5 d^{2} 6 p$ & $5 / 2$ & 0.17 & $8.95(9)$ \\
\hline 104.9200 & 16204.85 & $5 d^{3}$ & $9 / 2$ & 111515.16 & $5 d^{2} 6 p$ & $7 / 2$ & 0.42 & $1.59(10)$ \\
\hline 105.0920 & 3537.49 & $5 d^{3}$ & $5 / 2$ & 98691.76 & $5 d^{2} 6 p$ & $5 / 2$ & -0.25 & $3.43(9)$ \\
\hline 105.1511 & 18519.01 & $5 d^{3}$ & $3 / 2$ & 113620.96 & $5 d^{2} 6 p$ & $5 / 2$ & -0.81 & $9.30(8)$ \\
\hline 105.4802 & 28412.13 & $5 d^{3}$ & $7 / 2$ & 123216.93 & $5 d^{2} 6 p$ & $5 / 2$ & -0.35 & $2.68(9)$ \\
\hline 105.8243 & 18364.76 & $5 d^{3}$ & $1 / 2$ & 112861.20 & $5 d^{2} 6 p$ & $1 / 2$ & -0.52 & $1.78(9)$ \\
\hline 105.9970 & 18519.01 & $5 d^{3}$ & $3 / 2$ & 112861.20 & $5 d^{2} 6 p$ & $1 / 2$ & -0.73 & $1.09(9)$ \\
\hline 106.0164 & 17189.88 & $5 d^{3}$ & $5 / 2$ & 111515.16 & $5 d^{2} 6 p$ & $7 / 2$ & -0.74 & $1.09(9)$ \\
\hline 106.3286 & 16204.85 & $5 d^{3}$ & $9 / 2$ & 110252.75 & $5 d^{2} 6 p$ & $9 / 2$ & 0.17 & $8.65(9)$ \\
\hline 106.6873 & 10220.65 & $5 d^{3}$ & $3 / 2$ & 103952.54 & $5 d^{2} 6 p$ & $3 / 2$ & -0.59 & $1.52(9)$ \\
\hline 106.7742 & 22766.37 & $5 d^{3}$ & $5 / 2$ & 116421.86 & $5 d^{2} 6 p$ & $3 / 2$ & -0.11 & $4.53(9)$ \\
\hline $106.8247^{c}$ & 9256.39 & $5 d^{3}$ & $9 / 2$ & 102867.63 & $5 d^{2} 6 p$ & $7 / 2$ & 0.20 & $9.22(9)$ \\
\hline $106.8247^{c}$ & 15260.92 & $5 d^{3}$ & $7 / 2$ & 108871.96 & $5 d^{2} 6 p$ & $7 / 2$ & -0.51 & $1.79(9)$ \\
\hline 106.8628 & 6744.63 & $5 d^{3}$ & $7 / 2$ & 100322.36 & $5 d^{2} 6 p$ & $7 / 2$ & 0.09 & $7.24(9)$ \\
\hline 107.2597 & 28412.13 & $5 d^{3}$ & $7 / 2$ & 121644.05 & $5 d^{2} 6 p$ & $7 / 2$ & -0.61 & $1.42(9)$ \\
\hline 107.2963 & 22223.06 & $5 d^{3}$ & $11 / 2$ & 115422.93 & $5 d^{2} 6 p$ & $9 / 2$ & 0.57 & $2.14(10)$ \\
\hline 107.8839 & 17189.88 & $5 d^{3}$ & $5 / 2$ & 109882.34 & $5 d^{2} 6 p$ & $3 / 2$ & -0.79 & $9.45(8)$ \\
\hline 108.0410 & 25167.81 & $5 d^{3}$ & $3 / 2$ & 117725.20 & $5 d^{2} 6 p$ & $1 / 2$ & -0.40 & $2.27(9)$ \\
\hline 108.3616 & 6744.63 & $5 d^{3}$ & $7 / 2$ & 99028.26 & $5 d^{2} 6 p$ & $9 / 2$ & -0.48 & $1.90(9)$ \\
\hline 108.4838 & 11772.92 & $5 d^{3}$ & $1 / 2$ & 103952.54 & $5 d^{2} 6 p$ & $3 / 2$ & -0.96 & $6.19(8)$ \\
\hline 108.6786 & 24096.03 & $5 d^{3}$ & $9 / 2$ & 116110.43 & $5 d^{2} 6 p$ & $7 / 2$ & -0.25 & $3.20(9)$ \\
\hline 108.8026 & 11772.92 & $5 d^{3}$ & $1 / 2$ & 103682.37 & $5 d^{2} 6 p$ & $1 / 2$ & -0.67 & $1.22(9)$ \\
\hline
\end{tabular}


Table 3. (Continued.)

\begin{tabular}{|c|c|c|c|c|c|c|c|c|}
\hline \multirow[b]{2}{*}{$\lambda^{\mathrm{a}}(\mathrm{nm})$} & \multicolumn{3}{|c|}{ Lower level $^{\mathrm{a}}$} & \multicolumn{3}{|c|}{ Upper level ${ }^{\mathrm{a}}$} & \multirow[b]{2}{*}{$\log g f^{\mathrm{d}}$} & \multirow[b]{2}{*}{$g A^{\mathrm{d}}\left(\mathrm{s}^{-1}\right)$} \\
\hline & $E\left(\mathrm{~cm}^{-1}\right)$ & Configuration & $J$ & $E\left(\mathrm{~cm}^{-1}\right)$ & Configuration & $J$ & & \\
\hline 109.2687 & 18364.76 & $5 d^{3}$ & $1 / 2$ & 109882.34 & $5 d^{2} 6 p$ & $3 / 2$ & -0.64 & $1.27(9)$ \\
\hline 109.2973 & 15260.92 & $5 d^{3}$ & $7 / 2$ & 106754.40 & $5 d^{2} 6 p$ & $5 / 2$ & -0.53 & $1.67(9)$ \\
\hline 109.3259 & 15260.92 & $5 d^{3}$ & $7 / 2$ & 106730.40 & $5 d^{2} 6 p$ & $7 / 2$ & 0.03 & $6.02(9)$ \\
\hline 109.4527 & 18519.01 & $5 d^{3}$ & $3 / 2$ & 109882.34 & $5 d^{2} 6 p$ & $3 / 2$ & -0.29 & $2.84(9)$ \\
\hline 109.4970 & 24096.03 & $5 d^{3}$ & $9 / 2$ & 115422.93 & $5 d^{2} 6 p$ & $9 / 2$ & -0.37 & $2.37(9)$ \\
\hline 109.5511 & 28412.13 & $5 d^{3}$ & $7 / 2$ & 119694.03 & $5 d^{2} 6 p$ & $9 / 2$ & -0.82 & $8.45(8)$ \\
\hline 109.5844 & 25167.81 & $5 d^{3}$ & $3 / 2$ & 116421.86 & $5 d^{2} 6 p$ & $3 / 2$ & -0.60 & $1.39(9)$ \\
\hline 109.7651 & 27937.63 & $5 d^{3}$ & $5 / 2$ & 119041.11 & $5 d^{2} 6 p$ & $3 / 2$ & 0.03 & $5.89(9)$ \\
\hline 109.8239 & 10220.65 & $5 d^{3}$ & $3 / 2$ & 101275.42 & $5 d^{2} 6 p$ & $1 / 2$ & -0.45 & $1.96(9)$ \\
\hline 109.9046 & 3537.49 & $5 d^{3}$ & $5 / 2$ & 94525.28 & $5 d^{2} 6 p$ & $5 / 2$ & -0.20 & $3.52(9)$ \\
\hline 110.0660 & 22766.37 & $5 d^{3}$ & $5 / 2$ & 113620.96 & $5 d^{2} 6 p$ & $5 / 2$ & -0.43 & $2.03(9)$ \\
\hline 110.1725 & 22766.37 & $5 d^{3}$ & $5 / 2$ & 113533.11 & $5 d^{2} 6 p$ & $3 / 2$ & -1.00 & $5.44(8)$ \\
\hline 110.2937 & 28412.13 & $5 d^{3}$ & $7 / 2$ & 119079.04 & $5 d^{2} 6 p$ & $5 / 2$ & -0.04 & $5.03(9)$ \\
\hline 110.4269 & 18364.76 & $5 d^{3}$ & $1 / 2$ & 108922.34 & $5 d^{2} 6 p$ & $3 / 2$ & -0.65 & $1.22(9)$ \\
\hline 110.4659 & 16204.85 & $5 d^{3}$ & $9 / 2$ & 106730.40 & $5 d^{2} 6 p$ & $7 / 2$ & -0.84 & 7.89(8) \\
\hline 110.6149 & 18519.01 & $5 d^{3}$ & $3 / 2$ & 108922.34 & $5 d^{2} 6 p$ & $3 / 2$ & -0.44 & $1.97(9)$ \\
\hline 110.7341 & 45516.94 & $5 d^{2} 6 s$ & $1 / 2$ & 135823.21 & $5 d 6 s 6 p$ & $3 / 2$ & -0.60 & $1.37(9)$ \\
\hline 110.8284 & 10220.65 & $5 d^{3}$ & $3 / 2$ & 100449.92 & $5 d^{2} 6 p$ & $3 / 2$ & -0.44 & $1.96(9)$ \\
\hline 111.2430 & 0.00 & $5 d^{3}$ & $3 / 2$ & 89893.06 & $5 d^{2} 6 p$ & $3 / 2$ & -0.09 & $4.36(9)$ \\
\hline 111.3934 & 9256.39 & $5 d^{3}$ & $9 / 2$ & 99028.26 & $5 d^{2} 6 p$ & $9 / 2$ & -0.19 & $3.45(9)$ \\
\hline 111.5491 & 10220.65 & $5 d^{3}$ & $3 / 2$ & 99867.18 & $5 d^{2} 6 p$ & $3 / 2$ & -0.87 & 7.31(8) \\
\hline 111.9710 & 3537.49 & $5 d^{3}$ & $5 / 2$ & 92845.71 & $5 d^{2} 6 p$ & $7 / 2$ & -0.55 & $1.52(9)$ \\
\hline 113.0310 & 10220.65 & $5 d^{3}$ & $3 / 2$ & 98691.76 & $5 d^{2} 6 p$ & $5 / 2$ & -0.98 & $5.47(8)$ \\
\hline 113.0539 & 25167.81 & $5 d^{3}$ & $3 / 2$ & 113620.96 & $5 d^{2} 6 p$ & $5 / 2$ & -0.50 & $1.67(9)$ \\
\hline 113.3329 & 18519.01 & $5 d^{3}$ & $3 / 2$ & 106754.40 & $5 d^{2} 6 p$ & $5 / 2$ & -0.64 & $1.20(9)$ \\
\hline 113.5143 & 11772.92 & $5 d^{3}$ & $1 / 2$ & 99867.18 & $5 d^{2} 6 p$ & $3 / 2$ & -0.48 & $1.73(9)$ \\
\hline 113.5980 & 22223.06 & $5 d^{3}$ & $11 / 2$ & 110252.75 & $5 d^{2} 6 p$ & $9 / 2$ & -0.73 & $9.66(8)$ \\
\hline 113.6934 & 15260.92 & $5 d^{3}$ & $7 / 2$ & 103216.51 & $5 d^{2} 6 p$ & $5 / 2$ & -0.63 & $1.21(9)$ \\
\hline 113.7758 & 22766.37 & $5 d^{3}$ & $5 / 2$ & 110658.47 & $5 d^{2} 6 p$ & $5 / 2$ & -0.75 & $9.28(8)$ \\
\hline 114.0268 & 28412.13 & $5 d^{3}$ & $7 / 2$ & 116110.43 & $5 d^{2} 6 p$ & $7 / 2$ & -0.14 & $3.71(9)$ \\
\hline 114.3914 & 24096.03 & $5 d^{3}$ & $9 / 2$ & 111515.16 & $5 d^{2} 6 p$ & $7 / 2$ & -0.82 & 7.74(8) \\
\hline 114.7892 & 22766.37 & $5 d^{3}$ & $5 / 2$ & 109882.34 & $5 d^{2} 6 p$ & $3 / 2$ & -0.74 & $9.19(8)$ \\
\hline 115.0674 & 28412.13 & $5 d^{3}$ & $7 / 2$ & 115317.71 & $5 d^{2} 6 p$ & $5 / 2$ & -0.88 & $6.63(8)$ \\
\hline 115.2567 & 17189.88 & $5 d^{3}$ & $5 / 2$ & 103952.54 & $5 d^{2} 6 p$ & $3 / 2$ & -0.77 & $8.47(8)$ \\
\hline 115.3894 & 16204.85 & $5 d^{3}$ & $9 / 2$ & 102867.63 & $5 d^{2} 6 p$ & $7 / 2$ & -0.45 & $1.76(9)$ \\
\hline $116.1362^{\mathrm{c}}$ & 22766.37 & $5 d^{3}$ & $5 / 2$ & 108871.96 & $5 d^{2} 6 p$ & $7 / 2$ & -0.32 & $2.40(9)$ \\
\hline 116.2430 & 17189.88 & $5 d^{3}$ & $5 / 2$ & 103216.51 & $5 d^{2} 6 p$ & $5 / 2$ & -0.89 & $6.31(8)$ \\
\hline 116.7169 & 17189.88 & $5 d^{3}$ & $5 / 2$ & 102867.63 & $5 d^{2} 6 p$ & $7 / 2$ & -0.67 & $1.05(9)$ \\
\hline 116.9718 & 25167.81 & $5 d^{3}$ & $3 / 2$ & 110658.47 & $5 d^{2} 6 p$ & $5 / 2$ & -0.97 & $5.20(8)$ \\
\hline 117.2469 & 0.00 & $5 d^{3}$ & $3 / 2$ & 85289.99 & $5 d^{2} 6 p$ & $5 / 2$ & -0.69 & $9.92(8)$ \\
\hline 117.3590 & 28412.13 & $5 d^{3}$ & $7 / 2$ & 113620.96 & $5 d^{2} 6 p$ & $5 / 2$ & -0.97 & $5.21(8)$ \\
\hline 118.6169 & 10220.65 & $5 d^{3}$ & $3 / 2$ & 94525.28 & $5 d^{2} 6 p$ & $5 / 2$ & -0.41 & $1.85(9)$ \\
\hline 119.0982 & 22766.37 & $5 d^{3}$ & $5 / 2$ & 106730.40 & $5 d^{2} 6 p$ & $7 / 2$ & -0.98 & 4.91(8) \\
\hline 119.3447 & 43286.60 & $5 d^{3}$ & $5 / 2$ & 127077.24 & $5 d^{2} 6 p$ & $5 / 2$ & -0.36 & $2.06(9)$ \\
\hline 119.9961 & 43741.52 & $5 d^{3}$ & $3 / 2$ & 127077.24 & $5 d^{2} 6 p$ & $5 / 2$ & -0.50 & $1.49(9)$ \\
\hline 120.2898 & 17189.88 & $5 d^{3}$ & $5 / 2$ & 100322.36 & $5 d^{2} 6 p$ & $7 / 2$ & -0.48 & $1.53(9)$ \\
\hline 120.7871 & 43286.60 & $5 d^{3}$ & $5 / 2$ & 126076.70 & $5 d^{2} 6 p$ & $3 / 2$ & -0.42 & $1.75(9)$ \\
\hline 120.8885 & 27937.63 & $5 d^{3}$ & $5 / 2$ & 110658.47 & $5 d^{2} 6 p$ & $5 / 2$ & -0.52 & $1.36(9)$ \\
\hline 121.4545 & 43741.52 & $5 d^{3}$ & $3 / 2$ & 126076.70 & $5 d^{2} 6 p$ & $3 / 2$ & -0.74 & $8.13(8)$ \\
\hline 122.5690 & 25167.81 & $5 d^{3}$ & $3 / 2$ & 106754.40 & $5 d^{2} 6 p$ & $5 / 2$ & -0.54 & $1.29(9)$ \\
\hline 123.5568 & 27937.63 & $5 d^{3}$ & $5 / 2$ & 108871.96 & $5 d^{2} 6 p$ & $7 / 2$ & -0.73 & $8.18(8)$ \\
\hline 124.0360 & 43286.60 & $5 d^{3}$ & $5 / 2$ & 123908.20 & $5 d^{2} 6 p$ & $7 / 2$ & -0.30 & $2.19(9)$ \\
\hline 125.1095 & 43286.60 & $5 d^{3}$ & $5 / 2$ & 123216.93 & $5 d^{2} 6 p$ & $5 / 2$ & -0.85 & $6.07(8)$ \\
\hline 125.8253 & 43741.52 & $5 d^{3}$ & $3 / 2$ & 123216.93 & $5 d^{2} 6 p$ & $5 / 2$ & -0.67 & $9.08(8)$ \\
\hline 126.9155 & 27937.63 & $5 d^{3}$ & $5 / 2$ & 106730.40 & $5 d^{2} 6 p$ & $7 / 2$ & -0.83 & $6.09(8)$ \\
\hline 127.6453 & 28412.13 & $5 d^{3}$ & $7 / 2$ & 106754.40 & $5 d^{2} 6 p$ & $5 / 2$ & -0.85 & $5.78(8)$ \\
\hline 130.1214 & 28412.13 & $5 d^{3}$ & $7 / 2$ & 105263.40 & $5 d^{2} 6 p$ & $9 / 2$ & -0.79 & $6.41(8)$ \\
\hline 130.1992 & 22223.06 & $5 d^{3}$ & $11 / 2$ & 99028.26 & $5 d^{2} 6 p$ & $9 / 2$ & -0.99 & $4.02(8)$ \\
\hline 130.3107 & 49337.04 & $5 d^{2} 6 s$ & $5 / 2$ & 126076.70 & $5 d^{2} 6 p$ & $3 / 2$ & -0.86 & $5.40(8)$ \\
\hline 131.9398 & 43286.60 & $5 d^{3}$ & $5 / 2$ & 119079.04 & $5 d^{2} 6 p$ & $5 / 2$ & -0.94 & $4.40(8)$ \\
\hline 132.0053 & 43286.60 & $5 d^{3}$ & $5 / 2$ & 119041.11 & $5 d^{2} 6 p$ & $3 / 2$ & -0.77 & $6.53(8)$ \\
\hline 132.8391 & 27937.63 & $5 d^{3}$ & $5 / 2$ & 103216.51 & $5 d^{2} 6 p$ & $5 / 2$ & -0.80 & $5.92(8)$ \\
\hline
\end{tabular}


Table 3. (Continued.)

\begin{tabular}{|c|c|c|c|c|c|c|c|c|}
\hline \multirow[b]{2}{*}{$\lambda^{\mathrm{a}}(\mathrm{nm})$} & \multicolumn{3}{|c|}{ Lower level $^{\mathrm{a}}$} & \multicolumn{3}{|c|}{ Upper level $^{\mathrm{a}}$} & \multirow[b]{2}{*}{$\log g f^{\mathrm{d}}$} & \multirow[b]{2}{*}{$g A^{\mathrm{d}}\left(\mathrm{s}^{-1}\right)$} \\
\hline & $E\left(\mathrm{~cm}^{-1)}\right.$ & Configuration & $J$ & $E\left(\mathrm{~cm}^{-1}\right)$ & Configuration & $J$ & & \\
\hline 133.4574 & 27937.63 & $5 d^{3}$ & $5 / 2$ & 102867.63 & $5 d^{2} 6 p$ & $7 / 2$ & -0.75 & $6.60(8)$ \\
\hline 134.0638 & 39029.73 & $5 d^{2} 6 s$ & $7 / 2$ & 113620.96 & $5 d^{2} 6 p$ & $5 / 2$ & -0.53 & $1.11(9)$ \\
\hline 134.1000 & 49337.04 & $5 d^{2} 6 s$ & $5 / 2$ & 123908.20 & $5 d^{2} 6 p$ & $7 / 2$ & -0.86 & $5.15(8)$ \\
\hline 134.3080 & 28412.13 & $5 d^{3}$ & $7 / 2$ & 102867.63 & $5 d^{2} 6 p$ & $7 / 2$ & -0.82 & $5.60(8)$ \\
\hline 135.3545 & 49337.04 & $5 d^{2} 6 s$ & $5 / 2$ & 123216.93 & $5 d^{2} 6 p$ & $5 / 2$ & -0.73 & $6.77(8)$ \\
\hline 135.4484 & 35093.78 & $5 d^{3}$ & $5 / 2$ & 108922.34 & $5 d^{2} 6 p$ & $3 / 2$ & -0.34 & $1.68(9)$ \\
\hline 137.2007 & 43224.40 & $5 d^{2} 6 s$ & $9 / 2$ & 116110.43 & $5 d^{2} 6 p$ & $7 / 2$ & -0.58 & $9.27(8)$ \\
\hline 137.9578 & 39029.73 & $5 d^{2} 6 s$ & $7 / 2$ & 111515.16 & $5 d^{2} 6 p$ & $7 / 2$ & -0.38 & $1.46(9)$ \\
\hline 138.5070 & 43224.40 & $5 d^{2} 6 s$ & $9 / 2$ & 115422.93 & $5 d^{2} 6 p$ & $9 / 2$ & -0.07 & $2.96(9)$ \\
\hline 139.5466 & 35093.78 & $5 d^{2} 6 s$ & $5 / 2$ & 106754.40 & $5 d^{2} 6 p$ & $5 / 2$ & -0.48 & $1.15(9)$ \\
\hline 139.5935 & 35093.78 & $5 d^{2} 6 s$ & $5 / 2$ & 106730.40 & $5 d^{2} 6 p$ & $7 / 2$ & -0.84 & $4.99(8)$ \\
\hline 139.6088 & 39029.73 & $5 d^{2} 6 s$ & $7 / 2$ & 110658.47 & $5 d^{2} 6 p$ & $5 / 2$ & -0.68 & 7.09(8) \\
\hline 140.0568 & 47679.55 & $5 d^{2} 6 s$ & $3 / 2$ & 119079.04 & $5 d^{2} 6 p$ & $5 / 2$ & -0.54 & $9.81(8)$ \\
\hline 140.1314 & 47679.55 & $5 d^{2} 6 s$ & $3 / 2$ & 119041.11 & $5 d^{2} 6 p$ & $3 / 2$ & -0.62 & $8.20(8)$ \\
\hline 140.4036 & 39029.73 & $5 d^{2} 6 s$ & $7 / 2$ & 110252.75 & $5 d^{2} 6 p$ & $9 / 2$ & -0.76 & $5.86(8)$ \\
\hline 140.8649 & 32692.40 & $5 d^{2} 6 s$ & $3 / 2$ & 103682.37 & $5 d^{2} 6 p$ & $1 / 2$ & -0.43 & $1.25(9)$ \\
\hline 141.0338 & 45516.94 & $5 d^{2} 6 s$ & $1 / 2$ & 116421.86 & $5 d^{2} 6 p$ & $3 / 2$ & -0.67 & $7.19(8)$ \\
\hline 141.7953 & 32692.40 & $5 d^{2} 6 s$ & $3 / 2$ & 103216.51 & $5 d^{2} 6 p$ & $5 / 2$ & -0.70 & $6.53(8)$ \\
\hline 142.0678 & 52828.24 & $5 d^{2} 6 s$ & $7 / 2$ & 123216.93 & $5 d^{2} 6 p$ & $5 / 2$ & -0.39 & $1.34(9)$ \\
\hline 142.8101 & 46087.55 & $5 d^{2} 6 s$ & $5 / 2$ & 116110.43 & $5 d^{2} 6 p$ & $7 / 2$ & -0.28 & $1.72(9)$ \\
\hline 143.1797 & 39029.73 & $5 d^{2} 6 s$ & $7 / 2$ & 108871.96 & $5 d^{2} 6 p$ & $7 / 2$ & -0.66 & $7.16(8)$ \\
\hline 144.4456 & 46087.55 & $5 d^{2} 6 s$ & $5 / 2$ & 115317.71 & $5 d^{2} 6 p$ & $5 / 2$ & -0.50 & $9.99(8)$ \\
\hline 145.2249 & 35093.78 & $5 d^{2} 6 s$ & $5 / 2$ & 103952.54 & $5 d^{2} 6 p$ & $3 / 2$ & -0.99 & $3.21(8)$ \\
\hline 145.5503 & 58372.54 & $5 d^{2} 6 s$ & $7 / 2$ & 127077.24 & $5 d^{2} 6 p$ & $5 / 2$ & 0.14 & $4.38(9)$ \\
\hline 145.8088 & 32692.40 & $5 d^{2} 6 s$ & $3 / 2$ & 101275.42 & $5 d^{2} 6 p$ & $1 / 2$ & -0.95 & $3.59(8)$ \\
\hline 146.4327 & 43224.40 & $5 d^{2} 6 s$ & $9 / 2$ & 111515.16 & $5 d^{2} 6 p$ & $7 / 2$ & -0.40 & $1.25(9)$ \\
\hline $146.5662^{b}$ & 43286.60 & $5 d^{3}$ & $5 / 2$ & 111515.16 & $5 d^{2} 6 p$ & $7 / 2$ & -0.70 & $6.19(8)$ \\
\hline 146.7939 & 35093.78 & $5 d^{2} 6 s$ & $5 / 2$ & 103216.51 & $5 d^{2} 6 p$ & $5 / 2$ & -0.36 & $1.36(9)$ \\
\hline 147.0235 & 45516.94 & $5 d^{2} 6 s$ & $1 / 2$ & 113533.11 & $5 d^{2} 6 p$ & $3 / 2$ & -0.17 & $2.06(9)$ \\
\hline 147.5490 & 35093.78 & $5 d^{2} 6 s$ & $5 / 2$ & 102867.63 & $5 d^{2} 6 p$ & $7 / 2$ & 0.01 & $3.15(9)$ \\
\hline 147.5851 & 32692.40 & $5 d^{2} 6 s$ & $3 / 2$ & 100449.92 & $5 d^{2} 6 p$ & $3 / 2$ & -0.39 & $1.24(9)$ \\
\hline 147.6570 & 39029.73 & $5 d^{2} 6 s$ & $7 / 2$ & 106754.40 & $5 d^{2} 6 p$ & $5 / 2$ & -1.00 & $3.05(8)$ \\
\hline 147.7089 & 39029.73 & $5 d^{2} 6 s$ & $7 / 2$ & 106730.40 & $5 d^{2} 6 p$ & $7 / 2$ & -0.25 & 1.71(9) \\
\hline 147.8455 & 47679.55 & $5 d^{2} 6 s$ & $3 / 2$ & 115317.71 & $5 d^{2} 6 p$ & $5 / 2$ & 0.04 & $3.34(9)$ \\
\hline 148.0747 & 46087.55 & $5 d^{2} 6 s$ & $5 / 2$ & 113620.96 & $5 d^{2} 6 p$ & $5 / 2$ & -0.87 & $4.15(8)$ \\
\hline 148.7682 & 51860.40 & $5 d^{2} 6 s$ & $3 / 2$ & 119079.04 & $5 d^{2} 6 p$ & $5 / 2$ & -0.33 & $1.41(9)$ \\
\hline 148.8653 & 32692.40 & $5 d^{2} 6 s$ & $3 / 2$ & 99867.18 & $5 d^{2} 6 p$ & $3 / 2$ & -0.30 & $1.51(9)$ \\
\hline 149.1903 & 43224.40 & $5 d^{2} 6 s$ & $9 / 2$ & 110252.75 & $5 d^{2} 6 p$ & $9 / 2$ & -0.08 & $2.48(9)$ \\
\hline 149.5532 & 52828.24 & $5 d^{2} 6 s$ & $7 / 2$ & 119694.03 & $5 d^{2} 6 p$ & $9 / 2$ & -0.32 & $1.42(9)$ \\
\hline 149.5704 & 57050.05 & $5 d^{2} 6 s$ & $9 / 2$ & 123908.20 & $5 d^{2} 6 p$ & $7 / 2$ & 0.06 & $3.41(9)$ \\
\hline 149.6973 & 56415.54 & $5 d^{2} 6 s$ & $5 / 2$ & 123216.93 & $5 d^{2} 6 p$ & $5 / 2$ & -0.47 & $1.01(9)$ \\
\hline 149.7604 & 49337.04 & $5 d^{2} 6 s$ & $5 / 2$ & 116110.43 & $5 d^{2} 6 p$ & $7 / 2$ & 0.03 & $3.17(9)$ \\
\hline 150.9416 & 52828.24 & $5 d^{2} 6 s$ & $7 / 2$ & 119079.04 & $5 d^{2} 6 p$ & $5 / 2$ & -0.61 & 7.11(8) \\
\hline 150.9806 & 39029.73 & $5 d^{2} 6 s$ & $7 / 2$ & 105263.40 & $5 d^{2} 6 p$ & $9 / 2$ & 0.38 & 7.01(9) \\
\hline 151.5162 & 32692.40 & $5 d^{2} 6 s$ & $3 / 2$ & 98691.76 & $5 d^{2} 6 p$ & $5 / 2$ & -0.11 & $2.25(9)$ \\
\hline 151.5932 & 43224.40 & $5 d^{2} 6 s$ & $9 / 2$ & 109190.30 & $5 d^{2} 6 p$ & $11 / 2$ & 0.64 & $1.26(10)$ \\
\hline 151.6497 & 47679.55 & $5 d^{2} 6 s$ & $3 / 2$ & 113620.96 & $5 d^{2} 6 p$ & $5 / 2$ & -0.73 & $5.36(8)$ \\
\hline 151.8257 & 51860.40 & $5 d^{2} 6 s$ & $3 / 2$ & 117725.20 & $5 d^{2} 6 p$ & $1 / 2$ & -0.54 & $8.35(8)$ \\
\hline 151.8518 & 47679.55 & $5 d^{2} 6 s$ & $3 / 2$ & 113533.11 & $5 d^{2} 6 p$ & $3 / 2$ & -0.60 & $7.25(8)$ \\
\hline 151.9813 & 57050.05 & $5 d^{2} 6 s$ & $9 / 2$ & 122847.40 & $5 d^{2} 6 p$ & $11 / 2$ & 0.61 & $1.17(10)$ \\
\hline 152.0418 & 32692.40 & $5 d^{2} 6 s$ & $3 / 2$ & 98463.71 & $5 d^{2} 6 p$ & $1 / 2$ & -0.83 & $4.25(8)$ \\
\hline 152.3285 & 43224.40 & $5 d^{2} 6 s$ & $9 / 2$ & 108871.96 & $5 d^{2} 6 p$ & $7 / 2$ & -0.78 & $4.76(8)$ \\
\hline 152.5885 & 58372.54 & $5 d^{3}$ & $7 / 2$ & 123908.20 & $5 d^{2} 6 p$ & $7 / 2$ & 0.03 & $3.07(9)$ \\
\hline 152.8405 & 46087.55 & $5 d^{2} 6 s$ & $5 / 2$ & 111515.16 & $5 d^{2} 6 p$ & $7 / 2$ & -0.89 & $3.69(8)$ \\
\hline 153.0081 & 35093.78 & $5 d^{2} 6 s$ & $5 / 2$ & 100449.92 & $5 d^{2} 6 p$ & $3 / 2$ & -0.94 & $3.26(8)$ \\
\hline $153.3066^{c}$ & 56415.54 & $5 d^{2} 6 s$ & $5 / 2$ & 121644.05 & $5 d^{2} 6 p$ & $7 / 2$ & 0.36 & $6.48(9)$ \\
\hline $153.3066^{c}$ & 35093.78 & $5 d^{2} 6 s$ & $5 / 2$ & 100322.36 & $5 d^{2} 6 p$ & $7 / 2$ & -0.49 & $9.16(8)$ \\
\hline 153.4172 & 47679.55 & $5 d^{2} 6 s$ & $3 / 2$ & 112861.20 & $5 d^{2} 6 p$ & $1 / 2$ & -0.46 & $9.84(8)$ \\
\hline 154.8682 & 46087.55 & $5 d^{2} 6 s$ & $5 / 2$ & 110658.47 & $5 d^{2} 6 p$ & $5 / 2$ & -0.81 & $4.30(8)$ \\
\hline 154.8905 & 51860.40 & $5 d^{2} 6 s$ & $3 / 2$ & 116421.86 & $5 d^{2} 6 p$ & $3 / 2$ & -0.17 & $1.88(9)$ \\
\hline $155.3630^{\mathrm{b}}$ & 45516.94 & $5 d^{2} 6 s$ & $1 / 2$ & 109882.34 & $5 d^{2} 6 p$ & $3 / 2$ & -0.81 & $4.33(8)$ \\
\hline
\end{tabular}


Table 3. (Continued.)

\begin{tabular}{|c|c|c|c|c|c|c|c|c|}
\hline \multirow[b]{2}{*}{$\lambda^{\mathrm{a}}(\mathrm{nm})$} & \multicolumn{3}{|c|}{ Lower level $^{\mathrm{a}}$} & \multicolumn{3}{|c|}{ Upper level $^{\mathrm{a}}$} & \multirow[b]{2}{*}{$\log g f^{\mathrm{d}}$} & \multirow[b]{2}{*}{$g A^{\mathrm{d}}\left(\mathrm{s}^{-1}\right)$} \\
\hline & $E\left(\mathrm{~cm}^{-1}\right)$ & Configuration & $J$ & $E\left(\mathrm{~cm}^{-1}\right)$ & Configuration & $J$ & & \\
\hline 155.5596 & 49337.04 & $5 d^{2} 6 s$ & $5 / 2$ & 113620.96 & $5 d^{2} 6 p$ & $5 / 2$ & -0.60 & $6.87(8)$ \\
\hline 155.7952 & 39029.73 & $5 d^{2} 6 s$ & $7 / 2$ & 103216.51 & $5 d^{2} 6 p$ & $5 / 2$ & -0.55 & 7.64(8) \\
\hline 156.6463 & 39029.73 & $5 d^{2} 6 s$ & $7 / 2$ & 102867.63 & $5 d^{2} 6 p$ & $7 / 2$ & -0.60 & $6.85(8)$ \\
\hline 157.2377 & 35093.78 & $5 d^{2} 6 s$ & $5 / 2$ & 98691.76 & $5 d^{2} 6 p$ & $5 / 2$ & -0.47 & $9.19(8)$ \\
\hline $157.4654^{\mathrm{b}}$ & 43224.40 & $5 d^{2} 6 s$ & $9 / 2$ & 106730.40 & $5 d^{2} 6 p$ & $7 / 2$ & -0.95 & $2.99(8)$ \\
\hline 157.7148 & 45516.94 & $5 d^{2} 6 s$ & $1 / 2$ & 108922.34 & $5 d^{2} 6 p$ & $3 / 2$ & -0.98 & $2.79(8)$ \\
\hline 158.0219 & 52828.24 & $5 d^{2} 6 s$ & $7 / 2$ & 116110.43 & $5 d^{2} 6 p$ & $7 / 2$ & -0.60 & $6.68(8)$ \\
\hline 158.0490 & 58372.54 & $5 d^{2} 6 s$ & $7 / 2$ & 121644.05 & $5 d^{2} 6 p$ & $7 / 2$ & -0.50 & $8.58(8)$ \\
\hline 159.1471 & 46087.55 & $5 d^{2} 6 s$ & $5 / 2$ & 108922.34 & $5 d^{2} 6 p$ & $3 / 2$ & -0.50 & $8.42(8)$ \\
\hline 159.2746 & 46087.55 & $5 d^{2} 6 s$ & $5 / 2$ & 108871.96 & $5 d^{2} 6 p$ & $7 / 2$ & -0.35 & $1.18(9)$ \\
\hline 159.5825 & 56415.54 & $5 d^{2} 6 s$ & $5 / 2$ & 119079.04 & $5 d^{2} 6 p$ & $5 / 2$ & -0.28 & $1.36(9)$ \\
\hline 159.6317 & 57050.05 & $5 d^{2} 6 s$ & $9 / 2$ & 119694.03 & $5 d^{2} 6 p$ & $9 / 2$ & 0.15 & $3.65(9)$ \\
\hline 159.7573 & 52828.24 & $5 d^{2} 6 s$ & $7 / 2$ & 115422.9 & $5 d^{2} 6 p$ & $9 / 2$ & 0.09 & $3.24(9)$ \\
\hline 160.2657 & 64680.96 & $5 d^{2} 6 s$ & $3 / 2$ & 127077.24 & $5 d^{2} 6 p$ & $5 / 2$ & -0.77 & $4.43(8)$ \\
\hline 160.8282 & 49337.04 & $5 d^{2} 6 s$ & $5 / 2$ & 111515.16 & $5 d^{2} 6 p$ & $7 / 2$ & -0.34 & 1.19(9) \\
\hline 161.1613 & 59628.18 & $5 d^{2} 6 s$ & $1 / 2$ & 121677.79 & $5 d^{2} 6 p$ & $1 / 2$ & -0.87 & $3.47(8)$ \\
\hline 161.1893 & 43224.40 & $5 d^{2} 6 s$ & $9 / 2$ & 105263.40 & $5 d^{2} 6 p$ & $9 / 2$ & -0.44 & $9.28(8)$ \\
\hline $161.9156^{b}$ & 51860.40 & $5 d^{2} 6 s$ & $3 / 2$ & 113620.96 & $5 d^{2} 6 p$ & $5 / 2$ & -0.70 & $5.07(8)$ \\
\hline 162.8773 & 64680.96 & $5 d^{2} 6 s$ & $3 / 2$ & 126076.70 & $5 d^{2} 6 p$ & $3 / 2$ & -0.04 & $2.27(9)$ \\
\hline $163.0746^{\mathrm{c}}$ & 58372.54 & $5 d^{2} 6 s$ & $7 / 2$ & 119694.03 & $5 d^{2} 6 p$ & $9 / 2$ & 0.15 & $3.51(9)$ \\
\hline 163.1516 & 39029.73 & $5 d^{2} 6 s$ & $7 / 2$ & 100322.36 & $5 d^{2} 6 p$ & $7 / 2$ & -0.42 & $9.54(8)$ \\
\hline 163.2849 & 47679.55 & $5 d^{2} 6 s$ & $3 / 2$ & 108922.34 & $5 d^{2} 6 p$ & $3 / 2$ & -0.94 & $2.89(8)$ \\
\hline $163.9323^{b}$ & 51860.40 & $5 d^{2} 6 s$ & $3 / 2$ & 112861.20 & $5 d^{2} 6 p$ & $1 / 2$ & -0.78 & $4.11(8)$ \\
\hline 164.4933 & 52828.24 & $5 d^{2} 6 s$ & $7 / 2$ & 113620.96 & $5 d^{2} 6 p$ & $5 / 2$ & -0.28 & $1.30(9)$ \\
\hline 164.8999 & 46087.55 & $5 d^{2} 6 s$ & $5 / 2$ & 106730.40 & $5 d^{2} 6 p$ & $7 / 2$ & -0.08 & $2.06(9)$ \\
\hline 165.1658 & 49337.04 & $5 d^{2} 6 s$ & $5 / 2$ & 109882.34 & $5 d^{2} 6 p$ & $3 / 2$ & -0.56 & $6.72(8)$ \\
\hline $166.6491^{b}$ & 56415.54 & $5 d^{2} 6 s$ & $5 / 2$ & 116421.86 & $5 d^{2} 6 p$ & $3 / 2$ & -0.99 & $2.46(8)$ \\
\hline 166.6707 & 39029.73 & $5 d^{2} 6 s$ & $7 / 2$ & 99028.26 & $5 d^{2} 6 p$ & $9 / 2$ & -0.01 & 2.34(9) \\
\hline 167.6112 & 39029.73 & $5 d^{2} 6 s$ & $7 / 2$ & 98691.76 & $5 d^{2} 6 p$ & $5 / 2$ & -0.91 & 2.93(8) \\
\hline 167.6638 & 43224.40 & $5 d^{2} 6 s$ & $9 / 2$ & 102867.63 & $5 d^{2} 6 p$ & $7 / 2$ & -0.43 & $8.87(8)$ \\
\hline 167.9688 & 49337.04 & $5 d^{2} 6 s$ & $5 / 2$ & 108871.96 & $5 d^{2} 6 p$ & $7 / 2$ & -0.39 & $9.53(8)$ \\
\hline 168.2611 & 35093.78 & $5 d^{2} 6 s$ & $5 / 2$ & 94525.28 & $5 d^{2} 6 p$ & $5 / 2$ & -0.54 & $6.82(8)$ \\
\hline 168.3141 & 59628.18 & $5 d^{2} 6 s$ & $1 / 2$ & 119041.11 & $5 d^{2} 6 p$ & $3 / 2$ & -0.15 & $1.69(9)$ \\
\hline 169.3183 & 57050.05 & $5 d^{2} 6 s$ & $9 / 2$ & 116110.43 & $5 d^{2} 6 p$ & $7 / 2$ & -0.87 & $3.12(8)$ \\
\hline 169.7732 & 56415.54 & $5 d^{2} 6 s$ & $5 / 2$ & 115317.71 & $5 d^{2} 6 p$ & $5 / 2$ & -0.82 & $3.48(8)$ \\
\hline 170.0742 & 51860.40 & $5 d^{2} 6 s$ & $3 / 2$ & 110658.47 & $5 d^{2} 6 p$ & $5 / 2$ & -0.22 & $1.38(9)$ \\
\hline 170.3957 & 52828.24 & $5 d^{2} 6 s$ & $7 / 2$ & 111515.16 & $5 d^{2} 6 p$ & $7 / 2$ & -0.28 & $1.20(9)$ \\
\hline 170.8351 & 64680.96 & $5 d^{2} 6 s$ & $3 / 2$ & 123216.93 & $5 d^{2} 6 p$ & $5 / 2$ & -0.26 & $1.25(9)$ \\
\hline 171.1285 & 45516.94 & $5 d^{2} 6 s$ & $1 / 2$ & 103952.54 & $5 d^{2} 6 p$ & $3 / 2$ & -0.92 & $2.71(8)$ \\
\hline 171.3126 & 57050.05 & $5 d^{2} 6 s$ & $9 / 2$ & 115422.93 & $5 d^{2} 6 p$ & $9 / 2$ & -0.55 & $6.46(8)$ \\
\hline 171.9231 & 45516.94 & $5 d^{2} 6 s$ & $1 / 2$ & 103682.37 & $5 d^{2} 6 p$ & $1 / 2$ & -0.90 & $2.86(8)$ \\
\hline 172.1259 & 59628.18 & $5 d^{2} 6 s$ & $1 / 2$ & 117725.20 & $5 d^{2} 6 p$ & $1 / 2$ & -0.83 & $3.33(8)$ \\
\hline 172.8156 & 46087.55 & $5 d^{2} 6 s$ & $5 / 2$ & 103952.54 & $5 d^{2} 6 p$ & $3 / 2$ & -0.59 & $5.76(8)$ \\
\hline 173.1535 & 35093.78 & $5 d^{2} 6 s$ & $5 / 2$ & 92845.71 & $5 d^{2} 6 p$ & $7 / 2$ & 0.08 & $2.65(9)$ \\
\hline 173.1969 & 58372.54 & $5 d^{2} 6 s$ & $7 / 2$ & 116110.43 & $5 d^{2} 6 p$ & $7 / 2$ & -0.89 & $2.90(8)$ \\
\hline 174.1415 & 52828.24 & $5 d^{2} 6 s$ & $7 / 2$ & 110252.75 & $5 d^{2} 6 p$ & $9 / 2$ & -0.46 & $8.00(8)$ \\
\hline 174.1625 & 49337.04 & $5 d^{2} 6 s$ & $5 / 2$ & 106754.40 & $5 d^{2} 6 p$ & $5 / 2$ & -0.33 & $1.03(9)$ \\
\hline 174.8227 & 32692.40 & $5 d^{2} 6 s$ & $3 / 2$ & 89893.06 & $5 d^{2} 6 p$ & $3 / 2$ & -0.45 & 7.61(8) \\
\hline 175.0422 & 46087.55 & $5 d^{2} 6 s$ & $5 / 2$ & 103216.51 & $5 d^{2} 6 p$ & $5 / 2$ & -0.98 & $2.29(8)$ \\
\hline $175.0775^{b}$ & 56415.54 & $5 d^{2} 6 s$ & $5 / 2$ & 113533.11 & $5 d^{2} 6 p$ & $3 / 2$ & -0.63 & $5.06(8)$ \\
\hline 175.1365 & 43224.40 & $5 d^{2} 6 s$ & $9 / 2$ & 100322.36 & $5 d^{2} 6 p$ & $7 / 2$ & 0.05 & $2.41(9)$ \\
\hline 175.2833 & 58372.54 & $5 d^{2} 6 s$ & $7 / 2$ & 115422.93 & $5 d^{2} 6 p$ & $9 / 2$ & -0.08 & $1.80(9)$ \\
\hline 175.4475 & 64680.96 & $5 d^{2} 6 s$ & $3 / 2$ & 121677.79 & $5 d^{2} 6 p$ & $1 / 2$ & -0.68 & $4.52(8)$ \\
\hline 176.1182 & 46087.55 & $5 d^{2} 6 s$ & $5 / 2$ & 102867.63 & $5 d^{2} 6 p$ & $7 / 2$ & -0.63 & $5.05(8)$ \\
\hline 177.7052 & 47679.55 & $5 d^{2} 6 s$ & $3 / 2$ & 103952.54 & $5 d^{2} 6 p$ & $3 / 2$ & -0.31 & $1.03(9)$ \\
\hline 179.1987 & 43224.40 & $5 d^{2} 6 s$ & $9 / 2$ & 99028.26 & $5 d^{2} 6 p$ & $9 / 2$ & 0.14 & $2.88(9)$ \\
\hline 180.0605 & 47679.55 & $5 d^{2} 6 s$ & $3 / 2$ & 103216.51 & $5 d^{2} 6 p$ & $5 / 2$ & -0.52 & $6.17(8)$ \\
\hline 180.1947 & 39029.73 & $5 d^{2} 6 s$ & $7 / 2$ & 94525.28 & $5 d^{2} 6 p$ & $5 / 2$ & -0.08 & $1.73(9)$ \\
\hline 182.4848 & 35093.78 & $5 d^{2} 6 s$ & $5 / 2$ & 89893.06 & $5 d^{2} 6 p$ & $3 / 2$ & -0.27 & $1.06(9)$ \\
\hline 183.6041 & 57050.05 & $5 d^{2} 6 s$ & $9 / 2$ & 111515.16 & $5 d^{2} 6 p$ & $7 / 2$ & -0.45 & 7.05(8) \\
\hline $183.8300^{b}$ & 64680.96 & $5 d^{2} 6 s$ & $3 / 2$ & 119079.04 & $5 d^{2} 6 p$ & $5 / 2$ & -0.75 & $3.49(8)$ \\
\hline 183.9514 & 46087.55 & $5 d^{2} 6 s$ & $5 / 2$ & 100449.92 & $5 d^{2} 6 p$ & $3 / 2$ & -0.55 & $5.64(8)$ \\
\hline
\end{tabular}


Table 3. (Continued.)

\begin{tabular}{|c|c|c|c|c|c|c|c|c|}
\hline \multirow[b]{2}{*}{$\lambda^{\mathrm{a}}(\mathrm{nm})$} & \multicolumn{3}{|c|}{ Lower level $^{\mathrm{a}}$} & \multicolumn{3}{|c|}{ Upper level $^{\mathrm{a}}$} & \multirow[b]{2}{*}{$\log g f^{\mathrm{d}}$} & \multirow[b]{2}{*}{$g A^{\mathrm{d}}\left(\mathrm{s}^{-1}\right)$} \\
\hline & $E\left(\mathrm{~cm}^{-1}\right)$ & Configuration & $J$ & $E\left(\mathrm{~cm}^{-1}\right)$ & Configuration & $J$ & & \\
\hline 184.3559 & 56415.54 & $5 d^{2} 6 s$ & $5 / 2$ & 110658.47 & $5 d^{2} 6 p$ & $5 / 2$ & -0.38 & $8.25(8)$ \\
\hline 185.4391 & 52828.24 & $5 d^{2} 6 s$ & $7 / 2$ & 106754.40 & $5 d^{2} 6 p$ & $5 / 2$ & -0.71 & $3.82(8)$ \\
\hline 185.5213 & 52828.24 & $5 d^{2} 6 s$ & $7 / 2$ & 106730.40 & $5 d^{2} 6 p$ & $7 / 2$ & -0.33 & $9.15(8)$ \\
\hline 185.5997 & 49337.04 & $5 d^{2} 6 s$ & $5 / 2$ & 103216.51 & $5 d^{2} 6 p$ & $5 / 2$ & -0.43 & $7.11(8)$ \\
\hline 185.8182 & 39029.73 & $5 d^{2} 6 s$ & $7 / 2$ & 92845.71 & $5 d^{2} 6 p$ & $7 / 2$ & -0.10 & $1.55(9)$ \\
\hline 186.5815 & 47679.55 & $5 d^{2} 6 s$ & $3 / 2$ & 101275.42 & $5 d^{2} 6 p$ & $1 / 2$ & -0.74 & $3.49(8)$ \\
\hline 187.0317 & 56415.54 & $5 d^{2} 6 s$ & $5 / 2$ & 109882.34 & $5 d^{2} 6 p$ & $3 / 2$ & -0.22 & $1.14(9)$ \\
\hline 187.9601 & 57050.05 & $5 d^{2} 6 s$ & $9 / 2$ & 110252.75 & $5 d^{2} 6 p$ & $9 / 2$ & 0.04 & $2.05(9)$ \\
\hline 188.1728 & 58372.54 & $5 d^{2} 6 s$ & $7 / 2$ & 111515.16 & $5 d^{2} 6 p$ & $7 / 2$ & -0.63 & $4.48(8)$ \\
\hline 188.8687 & 45516.94 & $5 d^{2} 6 s$ & $1 / 2$ & 98463.71 & $5 d^{2} 6 p$ & $1 / 2$ & -0.45 & $6.59(8)$ \\
\hline 189.5004 & 47679.55 & $5 d^{2} 6 s$ & $3 / 2$ & 100449.92 & $5 d^{2} 6 p$ & $3 / 2$ & -1.00 & $1.86(8)$ \\
\hline 190.0987 & 46087.55 & $5 d^{2} 6 s$ & $5 / 2$ & 98691.76 & $5 d^{2} 6 p$ & $5 / 2$ & -0.59 & $4.71(8)$ \\
\hline 190.1225 & 32692.40 & $5 d^{2} 6 s$ & $3 / 2$ & 85289.99 & $5 d^{2} 6 p$ & $5 / 2$ & -0.02 & $1.80(9)$ \\
\hline 190.6355 & 56415.54 & $5 d^{2} 6 s$ & $5 / 2$ & 108871.96 & $5 d^{2} 6 p$ & $7 / 2$ & -0.91 & $2.27(8)$ \\
\hline 190.7116 & 52828.24 & $5 d^{2} 6 s$ & $7 / 2$ & 105263.40 & $5 d^{2} 6 p$ & $9 / 2$ & -0.59 & $4.77(8)$ \\
\hline 191.9672 & 51860.40 & $5 d^{2} 6 s$ & $3 / 2$ & 103952.54 & $5 d^{2} 6 p$ & $3 / 2$ & -0.93 & $2.12(8)$ \\
\hline 192.7521 & 58372.54 & $5 d^{2} 6 s$ & $7 / 2$ & 110252.75 & $5 d^{2} 6 p$ & $9 / 2$ & -0.20 & $1.13(9)$ \\
\hline $192.9684^{\mathrm{c}}$ & 57050.05 & $5 d^{2} 6 s$ & $9 / 2$ & 108871.96 & $5 d^{2} 6 p$ & $7 / 2$ & -0.43 & $6.58(8)$ \\
\hline 194.7189 & 51860.40 & $5 d^{2} 6 s$ & $3 / 2$ & 103216.51 & $5 d^{2} 6 p$ & $5 / 2$ & -0.95 & $1.98(8)$ \\
\hline 195.6454 & 49337.04 & $5 d^{2} 6 s$ & $5 / 2$ & 100449.92 & $5 d^{2} 6 p$ & $3 / 2$ & -0.76 & $3.03(8)$ \\
\hline 197.9017 & 49337.04 & $5 d^{2} 6 s$ & $5 / 2$ & 99867.18 & $5 d^{2} 6 p$ & $3 / 2$ & -0.63 & $4.01(8)$ \\
\hline 198.0220 & 58372.54 & $5 d^{2} 6 s$ & $7 / 2$ & 108871.96 & $5 d^{2} 6 p$ & $7 / 2$ & -0.31 & $8.30(8)$ \\
\hline 199.2181 & 35093.78 & $5 d^{2} 6 s$ & $5 / 2$ & 85289.99 & $5 d^{2} 6 p$ & $5 / 2$ & -0.41 & $6.55(8)$ \\
\hline 199.8421 & 52828.24 & $5 d^{2} 6 s$ & $7 / 2$ & 102867.63 & $5 d^{2} 6 p$ & $7 / 2$ & -0.86 & $2.29(8)$ \\
\hline 206.3848 & 46087.55 & $5 d^{2} 6 s$ & $5 / 2$ & 94525.28 & $5 d^{2} 6 p$ & $5 / 2$ & -0.59 & $4.04(8)$ \\
\hline 207.3454 & 57050.05 & $5 d^{2} 6 s$ & $9 / 2$ & 105263.40 & $5 d^{2} 6 p$ & $9 / 2$ & -0.62 & $3.70(8)$ \\
\hline 210.4861 & 52828.24 & $5 d^{2} 6 s$ & $7 / 2$ & 100322.36 & $5 d^{2} 6 p$ & $7 / 2$ & -0.38 & $6.31(8)$ \\
\hline 213.4649 & 51860.40 & $5 d^{2} 6 s$ & $3 / 2$ & 98691.76 & $5 d^{2} 6 p$ & $5 / 2$ & -0.79 & $2.38(8)$ \\
\hline 213.7992 & 46087.55 & $5 d^{2} 6 s$ & $5 / 2$ & 92845.71 & $5 d^{2} 6 p$ & $7 / 2$ & -0.88 & $1.91(8)$ \\
\hline 216.3819 & 52828.24 & $5 d^{2} 6 s$ & $7 / 2$ & 99028.26 & $5 d^{2} 6 p$ & $9 / 2$ & -0.31 & $7.03(8)$ \\
\hline 217.4297 & 64680.96 & $5 d^{2} 6 s$ & $3 / 2$ & 110658.47 & $5 d^{2} 6 p$ & $5 / 2$ & -0.78 & $2.36(8)$ \\
\hline 237.6076 & 64680.96 & $5 d^{2} 6 s$ & $3 / 2$ & 106754.40 & $5 d^{2} 6 p$ & $5 / 2$ & -0.70 & $2.35(8)$ \\
\hline
\end{tabular}

${ }^{a}$ From Kramida and Shirai (2009) except when otherwise indicated.

${ }^{\mathrm{b}}$ Wavelength calculated using the available experimental energy levels.

c Blended line.

d This work $\left(\mathrm{HFR}_{43}+\mathrm{CPOL}\right.$ calculations).

Table 4. Transition probabilities for forbidden lines in W IV. Only transitions for which $A$-values are greater than $5 \mathrm{~s}^{-1}$ and $\lambda$ shorter than $2000 \mathrm{~nm}$ are listed. $A(B)$ stands for $A \times 10^{B}$.

\begin{tabular}{|c|c|c|c|c|c|c|}
\hline \multirow[b]{2}{*}{$\lambda^{\mathrm{a}}(\mathrm{nm})$} & \multicolumn{2}{|c|}{ Lower level $^{\mathrm{b}}$} & \multicolumn{2}{|c|}{ Upper level $^{\mathrm{b}}$} & \multirow[b]{2}{*}{ Type } & \multirow[b]{2}{*}{$A^{\mathrm{c}}\left(\mathrm{s}^{-1}\right)$} \\
\hline & $E\left(\mathrm{~cm}^{-1}\right)$ & Designation & $E\left(\mathrm{~cm}^{-1}\right)$ & Designation & & \\
\hline 129.9519 & 10220.65 & $5 d^{3}{ }^{4} P_{3 / 2}$ & 87172.17 & $5 \mathrm{~d} 6 \mathrm{~s}^{2}{ }^{2} \mathrm{D}_{3 / 2}$ & $\mathrm{M} 1+\mathrm{E} 2$ & $6.93(+0)$ \\
\hline 138.9686 & 22766.37 & $5 d^{3}{ }^{2} D_{5 / 2}$ & 94725.05 & $5 \mathrm{~d} 6 \mathrm{~s}^{2}{ }^{2} \mathrm{D}_{5 / 2}$ & $\mathrm{M} 1+\mathrm{E} 2$ & $.07(+1)$ \\
\hline 139.0603 & 15260.92 & $5 d^{32} G_{7 / 2}$ & 87172.17 & $5 \mathrm{~d} 6 \mathrm{~s}^{2}{ }^{2} \mathrm{D}_{3 / 2}$ & E2 & $1.28(+1)$ \\
\hline 141.5849 & 24096.03 & $5 d^{32} G_{9 / 2}$ & 94725.05 & $5 \mathrm{~d} 6 \mathrm{~s}^{2}{ }^{2} \mathrm{D}_{5 / 2}$ & E2 & $8.93(+0)$ \\
\hline 143.7665 & 25167.81 & $5 d^{32} P_{3 / 2}$ & 94725.05 & $5 \mathrm{~d} 6 \mathrm{~s}^{2}{ }^{2} \mathrm{D}_{5 / 2}$ & $\mathrm{M} 1+\mathrm{E} 2$ & $9.55(+0)$ \\
\hline 145.6597 & 18519.01 & $5 d^{32} D_{3 / 2}$ & 87172.17 & $5 \mathrm{~d} 6 \mathrm{~s}^{2}{ }^{2} \mathrm{D}_{3 / 2}$ & $\mathrm{M} 1+\mathrm{E} 2$ & $8.83(+0)$ \\
\hline 149.7288 & 27937.63 & $5 d^{3}{ }^{2} F_{5 / 2}$ & 94725.05 & $5 \mathrm{~d} 6 \mathrm{~s}^{2}{ }^{2} \mathrm{D}_{5 / 2}$ & $\mathrm{M} 1+\mathrm{E} 2$ & $7.90(+0)$ \\
\hline 150.8002 & 28412.13 & $5 d^{32} \mathrm{~F}_{7 / 2}$ & 94725.05 & $5 \mathrm{~d} 6 \mathrm{~s}^{2}{ }^{2} \mathrm{D}_{5 / 2}$ & $\mathrm{M} 1+\mathrm{E} 2$ & $2.15(+1)$ \\
\hline 155.2655 & 22766.37 & $5 d^{32} D_{5 / 2}$ & 87172.17 & $5 \mathrm{~d} 6 \mathrm{~s}^{2}{ }^{2} \mathrm{D}_{3 / 2}$ & $\mathrm{M} 1+\mathrm{E} 2$ & $1.27(+1)$ \\
\hline 168.8204 & 27937.63 & $5 d^{3}{ }^{2} F_{5 / 2}$ & 87172.17 & $5 \mathrm{~d} 6 \mathrm{~s}^{2}{ }^{2} \mathrm{D}_{3 / 2}$ & $\mathrm{M} 1+\mathrm{E} 2$ & $2.98(+1)$ \\
\hline 169.2366 & 18519.01 & $5 d^{32} D_{3 / 2}$ & 77607.89 & $5 d^{2} 6 s^{2} S_{1 / 2}$ & E2 & $1.71(+1)$ \\
\hline 170.1837 & 28412.13 & $5 d^{3}{ }^{2} F_{7 / 2}$ & 87172.17 & $5 \mathrm{~d} 6 \mathrm{~s}^{2}{ }^{2} \mathrm{D}_{3 / 2}$ & E2 & $7.00(+0)$ \\
\hline 178.2827 & 3537.49 & $5 d^{34} F_{5 / 2}$ & 59628.18 & $5 d^{2} 6 s^{2} P_{1 / 2}$ & E2 & $7.91(+0)$ \\
\hline 179.5483 & 39029.73 & $5 d^{2} 6 s^{4} F_{7 / 2}$ & 94725.05 & $5 \mathrm{~d} 6 \mathrm{~s}^{2}{ }^{2} \mathrm{D}_{5 / 2}$ & $\mathrm{M} 1+\mathrm{E} 2$ & $1.64(+1)$ \\
\hline 182.3436 & 22766.37 & $5 d^{32} D_{5 / 2}$ & 77607.89 & $5 d^{2} 6 s^{2} S_{1 / 2}$ & E2 & $2.61(+1)$ \\
\hline 183.5544 & 32692.40 & $5 d^{2} 6 s^{4} F_{3 / 2}$ & 87172.17 & $5 \mathrm{~d} 6 \mathrm{~s}^{2}{ }^{2} \mathrm{D}_{3 / 2}$ & $\mathrm{M} 1+\mathrm{E} 2$ & $5.79(+0)$ \\
\hline
\end{tabular}


Table 4. (Continued.)

\begin{tabular}{|c|c|c|c|c|c|c|}
\hline \multirow[b]{2}{*}{$\lambda^{\mathrm{a}}(\mathrm{nm})$} & \multicolumn{2}{|c|}{ Lower level ${ }^{b}$} & \multicolumn{2}{|c|}{ Upper level $^{\mathrm{b}}$} & \multirow[b]{2}{*}{ Type } & \multirow[b]{2}{*}{$A^{\mathrm{c}}\left(\mathrm{s}^{-1}\right)$} \\
\hline & $E\left(\mathrm{~cm}^{-1}\right)$ & Designation & $E\left(\mathrm{~cm}^{-1}\right)$ & Designation & & \\
\hline 190.6938 & 25167.81 & $5 d^{32} \mathrm{P}_{3 / 2}$ & 77607.89 & $5 d^{2} 6 s^{2} S_{1 / 2}$ & $\mathrm{M} 1+\mathrm{E} 2$ & $2.40(+1)$ \\
\hline 192.0182 & 35093.78 & $5 d^{2} 6 s{ }^{4} F_{5 / 2}$ & 87172.17 & $5 \mathrm{~d} 6 \mathrm{~s}^{2}{ }^{2} \mathrm{D}_{3 / 2}$ & $\mathrm{M} 1+\mathrm{E} 2$ & $2.71(+1)$ \\
\hline 194.1723 & 43224.40 & $5 d^{2} 6 s{ }^{4} F_{9 / 2}$ & 94725.05 & $5 \mathrm{~d} 6 \mathrm{~s}^{2}{ }^{2} \mathrm{D}_{5 / 2}$ & E2 & $1.04(+1)$ \\
\hline 194.4071 & 43286.60 & $5 d^{32} D_{5 / 2}$ & 94725.05 & $5 \mathrm{~d} 6 \mathrm{~s}^{2}{ }^{2} \mathrm{D}_{5 / 2}$ & $\mathrm{M} 1+\mathrm{E} 2$ & $8.24(+0)$ \\
\hline 202.3331 & 10220.65 & $5 d^{3}{ }^{4} P_{3 / 2}$ & 59628.18 & $5 d^{2} 6 s^{2} P_{1 / 2}$ & E2 & $2.97(+1)$ \\
\hline 209.6669 & 0.00 & $5 d^{3}{ }^{4} F_{3 / 2}$ & 47679.55 & $5 d^{2} 6 s{ }^{4} P_{3 / 2}$ & $\mathrm{M} 1+\mathrm{E} 2$ & $8.03(+0)$ \\
\hline 218.2744 & 3537.49 & $5 d^{3}{ }^{4} F_{5 / 2}$ & 49337.04 & $5 d^{2} 6 s^{2} F_{5 / 2}$ & E2 & $6.73(+0)$ \\
\hline 219.6298 & 0.00 & $5 d^{3}{ }^{4} F_{3 / 2}$ & 45516.94 & $5 d^{2} 6 s^{4} P_{1 / 2}$ & E2 & $6.11(+1)$ \\
\hline 220.2538 & 49337.04 & $5 d^{2} 6 s^{2} F_{5 / 2}$ & 94725.05 & $5 \mathrm{~d} 6 \mathrm{~s}^{2}{ }^{2} \mathrm{D}_{5 / 2}$ & $\mathrm{M} 1+\mathrm{E} 2$ & $3.23(+1)$ \\
\hline 226.4712 & 3537.49 & $5 d^{34} F_{5 / 2}$ & 47679.55 & $5 d^{2} 6 s^{4} P_{3 / 2}$ & $\mathrm{M} 1+\mathrm{E} 2$ & $3.01(+1)$ \\
\hline 227.7950 & 43286.60 & $5 d^{3}{ }^{2} D_{5 / 2}$ & 87172.17 & $5 \mathrm{~d} 6 \mathrm{~s}^{2}{ }^{2} \mathrm{D}_{3 / 2}$ & $\mathrm{M} 1+\mathrm{E} 2$ & $9.32(+0)$ \\
\hline 229.4353 & 9256.39 & $5 d^{3}{ }^{4} F_{9 / 2}$ & 52828.24 & $5 d^{2} 6 s^{2} F_{7 / 2}$ & $\mathrm{M} 1+\mathrm{E} 2$ & $1.13(+1)$ \\
\hline 231.8848 & 15260.92 & $5 d^{32} G_{7 / 2}$ & 58372.54 & $5 d^{2} 6 s^{2} G_{7 / 2}$ & $\mathrm{M} 1+\mathrm{E} 2$ & $1.07(+1)$ \\
\hline 234.7117 & 6744.63 & $5 d^{3}{ }^{4} F_{7 / 2}$ & 49337.04 & $5 d^{2} 6 s^{2} F_{5 / 2}$ & $\mathrm{M} 1+\mathrm{E} 2$ & $1.59(+1)$ \\
\hline 237.0760 & 16204.85 & $5 d^{3}{ }^{2} \mathrm{H}_{9 / 2}$ & 58372.54 & $5 d^{2} 6 s^{2} G_{7 / 2}$ & E2 & $1.29(+1)$ \\
\hline 238.1391 & 3537.49 & $5 d^{3}{ }^{4} F_{5 / 2}$ & 45516.94 & $5 d^{2} 6 s{ }^{4} P_{1 / 2}$ & E2 & $2.86(+1)$ \\
\hline 238.5077 & 22766.37 & $5 d^{32} D_{5 / 2}$ & 64680.96 & $5 d^{2} 6 s^{2} P_{3 / 2}$ & E2 & $1.22(+1)$ \\
\hline 238.6089 & 52828.24 & $5 d^{2} 6 s^{2} F_{7 / 2}$ & 94725.05 & $5 \mathrm{~d} 6 \mathrm{~s}^{2}{ }^{2} \mathrm{D}_{5 / 2}$ & E2 & $.33(+1)$ \\
\hline 239.2238 & 15260.92 & $5 d^{32} G_{7 / 2}$ & 57050.05 & $5 d^{2} 6 s^{2} G_{9 / 2}$ & $\mathrm{M} 1+\mathrm{E} 2$ & $6.31(+0)$ \\
\hline 242.9124 & 15260.92 & $5 d^{32} G_{7 / 2}$ & 56415.54 & $5 d^{2} 6 s^{2} D_{5 / 2}$ & $\mathrm{M} 1+\mathrm{E} 2$ & $1.37(+1)$ \\
\hline 243.1809 & 18519.01 & $5 d^{32} D_{3 / 2}$ & 59628.18 & $5 d^{2} 6 s^{2} P_{1 / 2}$ & $\mathrm{M} 1+\mathrm{E} 2$ & $1.15(+1)$ \\
\hline 243.3263 & 46087.55 & $5 d^{2} 6 s^{4} P_{5 / 2}$ & 87172.17 & $5 \mathrm{~d} 6 \mathrm{~s}^{2}{ }^{2} \mathrm{D}_{3 / 2}$ & $\mathrm{M} 1+\mathrm{E} 2$ & $8.03(+1)$ \\
\hline 244.2162 & 6744.63 & $5 d^{34} F_{7 / 2}$ & 47679.55 & $5 d^{2} 6 s^{4} P_{3 / 2}$ & E2 & $3.18(+1)$ \\
\hline 244.7527 & 16204.85 & $5 d^{3}{ }^{2} H_{9 / 2}$ & 57050.05 & $5 d^{2} 6 s^{2} G_{9 / 2}$ & $\mathrm{M} 1+\mathrm{E} 2$ & $2.31(+1)$ \\
\hline 249.3792 & 11772.92 & $5 d^{3}{ }^{4} P_{1 / 2}$ & 51860.40 & $5 d^{2} 6 s^{2} D_{3 / 2}$ & $\mathrm{M} 1+\mathrm{E} 2$ & $5.60(+0)$ \\
\hline 249.4217 & 9256.39 & $5 d^{3}{ }^{4} F_{9 / 2}$ & 49337.04 & $5 d^{2} 6 s^{2} F_{5 / 2}$ & E2 & $5.89(+0)$ \\
\hline 253.0042 & 25167.81 & $5 d^{3}{ }^{2} P_{3 / 2}$ & 64680.96 & $5 d^{2} 6 s^{2} P_{3 / 2}$ & E2 & $1.61(+1)$ \\
\hline 260.9539 & 56415.54 & $5 d^{2} 6 s^{2} D_{5 / 2}$ & 94725.05 & $5 \mathrm{~d} 6 \mathrm{~s}^{2}{ }^{2} \mathrm{D}_{5 / 2}$ & $\mathrm{M} 1+\mathrm{E} 2$ & $1.82(+1)$ \\
\hline 262.7283 & 18364.76 & $5 \mathrm{~d}^{3}{ }^{2} \mathrm{P}_{1 / 2}$ & 56415.54 & $5 d^{2} 6 s^{2} D_{5 / 2}$ & E2 & $8.15(+0)$ \\
\hline 263.7978 & 18519.01 & $5 d^{32} D_{3 / 2}$ & 56415.54 & $5 d^{2} 6 s^{2} D_{5 / 2}$ & $\mathrm{M} 1+\mathrm{E} 2$ & $1.14(+1)$ \\
\hline 264.2259 & 49337.04 & $5 d^{2} 6 s^{2} F_{5 / 2}$ & 87172.17 & $5 \mathrm{~d} 6 \mathrm{~s}^{2}{ }^{2} \mathrm{D}_{3 / 2}$ & E2 & $4.19(+1)$ \\
\hline 265.3490 & 57050.05 & $5 d^{2} 6 s^{2} G_{5 / 2}$ & 94725.05 & $5 \mathrm{~d} 6 \mathrm{~s}^{2}{ }^{2} \mathrm{D}_{5 / 2}$ & E2 & $5.19(+1)$ \\
\hline 266.1096 & 15260.92 & $5 d^{32} G_{7 / 2}$ & 52828.24 & $5 d^{2} 6 s^{2} F_{7 / 2}$ & $\mathrm{M} 1+\mathrm{E} 2$ & $8.78(+0)$ \\
\hline 266.1323 & 11772.92 & $5 d^{3}{ }^{4} P_{1 / 2}$ & 49337.04 & $5 d^{2} 6 s^{2} F_{5 / 2}$ & E2 & $8.33(+0)$ \\
\hline 266.8799 & 10220.65 & $5 d^{3}{ }^{4} P_{3 / 2}$ & 47679.55 & $5 d^{2} 6 s^{4} P_{3 / 2}$ & $\mathrm{M} 1+\mathrm{E} 2$ & $7.26(+0)$ \\
\hline 271.4288 & 9256.39 & $5 d^{3}{ }^{4} F_{9 / 2}$ & 46087.55 & $5 d^{2} 6 s^{4} P_{5 / 2}$ & E2 & $2.43(+1)$ \\
\hline 272.9687 & 16204.85 & $5 d^{32} \mathrm{H}_{9 / 2}$ & 52828.24 & $5 d^{2} 6 s^{2} F_{7 / 2}$ & $\mathrm{M} 1+\mathrm{E} 2$ & $1.50(+1)$ \\
\hline 273.1471 & 15260.92 & $5 d^{3}{ }^{2} G_{7 / 2}$ & 51860.40 & $5 d^{2} 6 s^{2} D_{3 / 2}$ & E2 & $4.08(+1)$ \\
\hline 274.0435 & 6744.63 & $5 d^{3}{ }^{4} F_{7 / 2}$ & 43224.40 & $5 d^{2} 6 s^{4} F_{9 / 2}$ & E2 & $1.25(+1)$ \\
\hline 275.0029 & 58372.54 & $5 d^{2} 6 s^{2} G_{7 / 2}$ & 94725.05 & $5 \mathrm{~d} 6 \mathrm{~s}^{2}{ }^{2} \mathrm{D}_{5 / 2}$ & E2 & $4.47(+1)$ \\
\hline 275.6374 & 28412.13 & $5 \mathrm{~d}^{3}{ }^{2} \mathrm{~F}_{7 / 2}$ & 64680.96 & $5 d^{2} 6 s^{2} P_{3 / 2}$ & E2 & $3.58(+1)$ \\
\hline 276.5475 & 22223.06 & $5 d^{32} \mathrm{H}_{11 / 2}$ & 58372.54 & $5 d^{2} 6 s^{2} G_{7 / 2}$ & E2 & $1.18(+1)$ \\
\hline 278.7264 & 10220.65 & $5 d^{34} P_{3 / 2}$ & 46087.55 & $5 d^{2} 6 s{ }^{4} P_{5 / 2}$ & $\mathrm{M} 1+\mathrm{E} 2$ & $1.35(+1)$ \\
\hline 281.6688 & 3537.49 & $5 d^{3}{ }^{4} F_{5 / 2}$ & 39029.73 & $5 d^{2} 6 s^{4} F_{7 / 2}$ & E2 & $1.60(+1)$ \\
\hline 283.1084 & 51860.40 & $5 d^{2} 6 s^{2} D_{3 / 2}$ & 87172.17 & $5 \mathrm{~d} 6 \mathrm{~s}^{2}{ }^{2} \mathrm{D}_{3 / 2}$ & E2 & $1.59(+1)$ \\
\hline 284.8420 & 59628.18 & $5 d^{2} 6 s^{2} P_{1 / 2}$ & 94725.05 & $5 \mathrm{~d} 6 \mathrm{~s}^{2}{ }^{2} \mathrm{D}_{5 / 2}$ & E2 & $5.88(+0)$ \\
\hline 284.8671 & 0.00 & $5 d^{3}{ }^{4} \mathrm{~F}_{3 / 2}$ & 35093.78 & $5 d^{2} 6 s^{4} F_{5 / 2}$ & E2 & $1.58(+1)$ \\
\hline 287.0494 & 22223.06 & $5 \mathrm{~d}^{32} \mathrm{H}_{11 / 2}$ & 57050.05 & $5 d^{2} 6 s^{2} G_{9 / 2}$ & $\mathrm{M} 1+\mathrm{E} 2$ & $3.61(+1)$ \\
\hline 291.0870 & 52828.24 & $5 d^{2} 6 s^{2} F_{70}$ & 87172.17 & $5 \mathrm{~d} 6 \mathrm{~s}^{2}{ }^{2} \mathrm{D}_{3 / 2}$ & E2 & $3.70(+1)$ \\
\hline 291.2790 & 43286.60 & $5 d^{32} D_{5 / 2}$ & 77607.89 & $5 d^{2} 6 s^{2} S_{1 / 2}$ & E2 & $2.47(+1)$ \\
\hline 291.6596 & 24096.03 & $5 d^{32} G_{9 / 2}$ & 58372.54 & $5 d^{2} 6 s^{2} G_{7 / 2}$ & $\mathrm{M} 1+\mathrm{E} 2$ & $4.05(+1)$ \\
\hline 293.3748 & 15260.92 & $5 d^{32} G_{7 / 2}$ & 49337.04 & $5 d^{2} 6 s^{2} F_{5 / 2}$ & $\mathrm{M} 1+\mathrm{E} 2$ & $9.91(+0)$ \\
\hline 294.3086 & 9256.39 & $5 d^{3}{ }^{4} F_{9 / 2}$ & 43224.40 & $5 d^{2} 6 s{ }^{4} F_{9 / 2}$ & $\mathrm{M} 1+\mathrm{E} 2$ & $2.63(+1)$ \\
\hline 295.1919 & 43741.52 & $5 d^{32} D_{3 / 2}$ & 77607.89 & $5 d^{2} 6 s^{2} S_{1 / 2}$ & E2 & $2.97(+1)$ \\
\hline 297.0974 & 22766.37 & $5 d^{32} D_{5 / 2}$ & 56415.54 & $5 d^{2} 6 s^{2} D_{5 / 2}$ & $\mathrm{M} 1+\mathrm{E} 2$ & $1.48(+1)$ \\
\hline 301.7334 & 16204.85 & $5 d^{32} H_{9 / 2}$ & 49337.04 & $5 d^{2} 6 s^{2} F_{5 / 2}$ & E2 & $2.01(+1)$ \\
\hline 305.7926 & 0.00 & $5 d^{3}{ }^{4} F_{3 / 2}$ & 32692.40 & $5 d^{2} 6 s^{4} F_{3 / 2}$ & $\mathrm{M} 1+\mathrm{E} 2$ & $1.48(+1)$ \\
\hline 309.3208 & 24096.03 & $5 d^{3}{ }^{2} G_{9 / 2}$ & 56415.54 & $5 d^{2} 6 s^{2} D_{5 / 2}$ & E2 & $1.79(+1)$ \\
\hline 309.6505 & 6744.63 & $5 d^{3}{ }^{4} F_{7 / 2}$ & 39029.73 & $5 d^{2} 6 s^{4} F_{7 / 2}$ & E2 & $1.18(+1)$ \\
\hline 311.5240 & 45516.94 & $5 d^{2} 6 s{ }^{4} P_{1 / 2}$ & 77607.89 & $5 d^{2} 6 s^{2} S_{1 / 2}$ & M1 & $5.09(+0)$ \\
\hline 315.4601 & 27937.63 & $5 d^{3}{ }^{2} F_{5 / 2}$ & 59628.18 & $5 d^{2} 6 s^{2} P_{1 / 2}$ & E2 & $3.16(+1)$ \\
\hline 316.8024 & 3537.49 & $5 d^{3}{ }^{4} F_{5 / 2}$ & 35093.78 & $5 d^{2} 6 s^{4} F_{5 / 2}$ & $\mathrm{M} 1+\mathrm{E} 2$ & $7.33(+0)$ \\
\hline
\end{tabular}


Table 4. (Continued.)

\begin{tabular}{|c|c|c|c|c|c|c|}
\hline \multirow[t]{2}{*}{$\lambda^{\mathrm{a}}(\mathrm{nm})$} & \multicolumn{2}{|c|}{ Lower level $^{\mathrm{b}}$} & \multicolumn{2}{|c|}{ Upper level $^{\mathrm{b}}$} & \multirow[t]{2}{*}{ Type } & \multirow[t]{2}{*}{$A^{\mathrm{c}}\left(\mathrm{s}^{-1}\right)$} \\
\hline & $E\left(\mathrm{~cm}^{-1}\right)$ & Designation & $E\left(\mathrm{~cm}^{-1}\right)$ & Designation & & \\
\hline 324.3013 & 15260.92 & $5 d^{3}{ }^{2} G_{7 / 2}$ & 46087.55 & $5 d^{2} 6 s{ }^{4} P_{5 / 2}$ & $\mathrm{M} 1+\mathrm{E} 2$ & $5.36(+0)$ \\
\hline 326.6479 & 22223.06 & $5 d^{32} \mathrm{H}_{11 / 2}$ & 52828.24 & $5 d^{2} 6 s^{2} F_{7 / 2}$ & $\mathrm{E} 2$ & $2.73(+1)$ \\
\hline 327.8855 & 17189.88 & $5 d^{34} P_{5 / 2}$ & 47679.55 & $5 d^{2} 6 s^{4} P_{3 / 2}$ & E2 & $1.03(+1)$ \\
\hline 332.7484 & 64680.96 & $5 d^{2} 6 s^{2} P_{3 / 2}$ & 94725.05 & $5 \mathrm{~d} 6 \mathrm{~s}^{2}{ }^{2} \mathrm{D}_{5 / 2}$ & $\mathrm{M} 1+\mathrm{E} 2$ & $1.46(+1)$ \\
\hline 334.0354 & 47679.55 & $5 d^{2} 6 s^{4} P_{3 / 2}$ & 77607.89 & $5 d^{2} 6 s^{2} S_{1 / 2}$ & $\mathrm{M} 1+\mathrm{E} 2$ & $3.39(+1)$ \\
\hline 334.5456 & 16204.85 & $5 d^{32} \mathrm{H}_{9 / 2}$ & 46087.55 & $5 d^{2} 6 s^{4} P_{5 / 2}$ & E2 & $9.25(+0)$ \\
\hline 342.8971 & 3537.49 & $5 d^{3}{ }^{4} F_{5 / 2}$ & 32692.40 & $5 d^{2} 6 s^{4} F_{3 / 2}$ & $\mathrm{M} 1+\mathrm{E} 2$ & $8.80(+0)$ \\
\hline 347.1273 & 58372.54 & $5 d^{2} 6 s^{2} G_{7 / 2}$ & 87172.17 & $5 \mathrm{~d} 6 \mathrm{~s}^{2}{ }^{2} \mathrm{D}_{3 / 2}$ & $\mathrm{E} 2$ & $5.00(+0)$ \\
\hline 351.0158 & 15260.92 & $5 d^{32} G_{7 / 2}$ & 43741.52 & $5 d^{32} D_{3 / 2}$ & E2 & $5.60(+0)$ \\
\hline 352.6434 & 6744.63 & $5 d^{3}{ }^{4} \mathrm{~F}_{7 / 2}$ & 35093.78 & $5 d^{2} 6 s{ }^{4} F_{5 / 2}$ & M1+E2 & $5.93(+0)$ \\
\hline 352.9185 & 17189.88 & $5 d^{3}{ }^{4} P_{5 / 2}$ & 45516.94 & $5 d^{2} 6 s{ }^{4} P_{1 / 2}$ & E2 & $8.72(+0)$ \\
\hline 362.9521 & 59628.18 & $5 d^{2} 6 s^{2} P_{1 / 2}$ & 87172.17 & $5 \mathrm{~d} 6 \mathrm{~s}^{2}{ }^{2} \mathrm{D}_{3 / 2}$ & $\mathrm{M} 1+\mathrm{E} 2$ & $6.37(+0)$ \\
\hline 383.0812 & 17189.88 & $5 d^{3}{ }^{4} P_{5 / 2}$ & 43286.60 & $5 d^{3}{ }^{2} D_{5 / 2}$ & $\mathrm{M} 1+\mathrm{E} 2$ & $9.57(+0)$ \\
\hline 388.2773 & 51860.40 & $5 d^{2} 6 s^{2} D_{3 / 2}$ & 77607.89 & $5 d^{2} 6 s^{2} S_{1 / 2}$ & $\mathrm{M} 1+\mathrm{E} 2$ & $1.14(+1)$ \\
\hline 403.6394 & 18519.01 & $5 d^{3}{ }^{2} D_{3 / 2}$ & 43286.60 & $5 d^{32} D_{5 / 2}$ & $\mathrm{M} 1+\mathrm{E} 2$ & $9.20(+0)$ \\
\hline 476.6214 & 22766.37 & $5 d^{3}{ }^{2} D_{5 / 2}$ & 43741.52 & $5 d^{32} D_{3 / 2}$ & $\mathrm{M} 1+\mathrm{E} 2$ & $6.35(+0)$ \\
\hline 556.0281 & 59628.18 & $5 d^{2} 6 s^{2} P_{1 / 2}$ & 77607.89 & $5 d^{2} 6 s^{2} S_{1 / 2}$ & M1 & $1.02(+1)$ \\
\hline 623.9793 & 6744.63 & $5 d^{3}{ }^{4} F_{7 / 2}$ & 22766.37 & $5 d^{32} D_{5 / 2}$ & M1 & $6.58(+0)$ \\
\hline 632.5807 & 27937.63 & $5 d^{3}{ }^{2} F_{5 / 2}$ & 43741.52 & $5 d^{32} D_{3 / 2}$ & $\mathrm{M} 1+\mathrm{E} 2$ & $5.72(+0)$ \\
\hline 651.3296 & 27937.63 & $5 d^{3}{ }^{2} F_{5 / 2}$ & 43286.60 & $5 d^{32} D_{5 / 2}$ & $\mathrm{M} 1+\mathrm{E} 2$ & $7.03(+0)$ \\
\hline 667.3048 & 3537.49 & $5 d^{3}{ }^{4} F_{5 / 2}$ & 18519.01 & $5 d^{32} D_{3 / 2}$ & $\mathrm{M} 1+\mathrm{E} 2$ & $5.88(+0)$ \\
\hline 852.7584 & 3537.49 & $5 d^{34} F_{5 / 2}$ & 15260.92 & $5 d^{32} G_{7 / 2}$ & M1 & $5.66(+0)$ \\
\hline 1131.5380 & 15260.92 & $5 d^{32} G_{7 / 2}$ & 24096.03 & $5 d^{32} G_{9 / 2}$ & M1 & $5.12(+0)$ \\
\hline 1204.7277 & 10220.65 & $5 d^{3}{ }^{4} P_{3 / 2}$ & 18519.01 & $5 d^{3}{ }^{2} D_{3 / 2}$ & M1 & $7.25(+0)$ \\
\hline 1516.6127 & 11772.92 & $5 d^{3}{ }^{4} P_{1 / 2}$ & 18364.76 & $5 d^{32} P_{1 / 2}$ & M1 & $5.86(+0)$ \\
\hline
\end{tabular}

a Vacuum $(\lambda<200 \mathrm{~nm})$ and air $(\lambda>200 \mathrm{~nm})$ wavelengths deduced from the experimental levels of Kramida and Shirai (2009).

${ }^{\mathrm{b}}$ From Kramida and Shirai (2009).

${ }^{c}$ This work (HFR ${ }_{43}+\mathrm{CPOL}$ calculations).

Unfortunately, as already mentioned, neither experimental nor theoretical radiative decay rates have been published so far for this ion. Nevertheless, in view of the discussion made in the previous section, the results obtained in this work are expected to be reliable in particular for the most intense lines for which the estimated accuracy is probably better than 10$15 \%$. Laboratory measurements are, however, strongly needed to confirm this assessment.

Forbidden lines also play an important role in plasma diagnostics because the corresponding radiation intensities are often very sensitive to electron temperature and density. Therefore, wavelengths and transition rates for such forbidden lines in various ionization stages of tungsten must be determined with high confidence. In table 1 , we present transition probabilities computed with our $\mathrm{HFR}_{43}+\mathrm{CPOL}$ model for selected magnetic dipole (M1) and electric quadrupole (E2) lines in W IV. When the two types of radiation contribute to the intensity of a line, the sum of both $A$-values is given.

\section{Conclusion}

Transition probabilities and oscillator strengths are reported for the first time for allowed and forbidden lines in a W IV ion. Their reliability has been assessed by the comparison of $\mathrm{HFR}+\mathrm{CPOL}$ and MCDF results and the reasonable convergence of the two sets of results. It is shown that the neglect of core-polarization effects can lead in this ion to lifetimes too short by about $15-20 \%$. HFR $_{43}+$ CPOL lifetimes appear systematically longer than $\mathrm{HFR}_{43}$ values, while $\mathrm{HFR}_{16}$ and $\mathrm{HFR}_{43}$ results agree generally well. Experimental lifetimes or transition probabilities in this ion would be most welcome to definitely assess the accuracy of this set of results and, eventually, to refine the present model.

\section{Acknowledgments}

EB and PQ are, respectively, the Research Director and the Senior Research Associate of the Belgian FRS-FNRS. Financial support from this organization and from ADAS-EU is acknowledged. SEY was financially supported by a grant from the FRS-FNRS (2011/V 6/5/013-IB/JN-1343) and from the Marien Ngouabi University, Congo and he is grateful to Belgian colleagues for their hospitality during his stay at Mons University.

\section{References}

Cowan R D 1981 The Theory of Atomic Structure and Spectra (Berkeley, CA: University of California Press)

Federici G 2001 Nucl. Fusion et al 411967

Fivet V, Biémont É, Engström L, Lundberg H, Nilsson H, Palmeri P and Quinet P 2008 J. Phys. B: At. Mol. Opt. Phys. 41015702

Fraga S, Karwowski J and Saxena K M S 1976 Handbook of Atomic Data (Amsterdam: Elsevier) 
Grant I P, McKenzie B J, Norrington P H, Mayers D F and Pyper N C 1980 Comput. Phys. Commun. 21207

Iglesias L, Kaufman V, Garcia-Riquelme O and Rico F R 1985 Phys. Scr. 31173

Kramida A E and Shirai T 2009 At. Data Nucl. Data Tables 95305

Lipschultz B et al 2001 J. Nucl. Mater. 290293286

McKenzie B J, Grant I P and Norrington P H 1980 Comput. Phys. Commun. 21233

Neu R et al 2005 Nucl. Fusion 45209

Nilsson H, Engström L, Lundberg H, Palmeri P, Fivet V, Quinet P and Biémont É 2008 Eur. Phys. J. D 4913

Norrington P H 2009 http://www.am.qub.ac.uk/DARC/
Palmeri P, Quinet P, Fivet V, Biémont É, Nilsson H, Engström L and Lundberg H 2008 Phys. Scr. 78015304

Pospieszczyk A 2006 Nuclear Fusion Research (Berlin: Springer) Quinet P, Palmeri P and Biémont É 2011 J. Phys. B: At. Mol. Opt. Phys. 44145005

Quinet P, Palmeri P, Biémont É, McCurdy M M, Rieger G, Pinnington E H, Wickliffe M E and Lawler J E 1999 Mon. Not. R. Astron. Soc. 307934

Quinet P, Vinogradoff V, Palmeri P and Biémont É 2010 J. Phys. B: At. Mol. Opt. Phys. 43144003

Skinner C H 2008 Can. J. Phys. 86285

Skinner C H 2009 Phys. Scr. T 134014022 